\title{
POINTWISE REGULARITY OF PARAMETERIZED AFFINE ZIPPER FRACTAL CURVES
}

\author{
BALÁZS BÁRÁNY, GERGELY KISS, AND ISTVÁN KOLOSSVÁRY
}

\begin{abstract}
We study the pointwise regularity of zipper fractal curves generated by affine mappings. Under the assumption of dominated splitting of index-1, we calculate the Hausdorff dimension of the level sets of the pointwise Hölder exponent for a subinterval of the spectrum. We give an equivalent characterization for the existence of regular pointwise Hölder exponent for Lebesgue almost every point. In this case, we extend the multifractal analysis to the full spectrum. In particular, we apply our results for de Rham's curve.
\end{abstract}

\section{Introduction and Statements}

Let us begin by recalling the general definition of fractal curves from Hutchinson [22] and Barnsley [3].

Definition 1.1. A system $\mathcal{S}=\left\{f_{0}, \ldots, f_{N-1}\right\}$ of contracting mappings of $\mathbb{R}^{d}$ to itself is called a zipper with vertices $Z=\left\{z_{0}, \ldots, z_{N}\right\}$ and signature $\varepsilon=$ $\left(\varepsilon_{0}, \ldots, \varepsilon_{N-1}\right), \varepsilon_{i} \in\{0,1\}$, if the cross-condition

$$
f_{i}\left(z_{0}\right)=z_{i+\varepsilon_{i}} \text { and } f_{i}\left(z_{N}\right)=z_{i+1-\varepsilon_{i}}
$$

holds for every $i=0, \ldots, N-1$. We call the system a self-affine zipper if the functions $f_{i}$ are affine contractive mappings of the form

$$
f_{i}(x)=A_{i} x+t_{i}, \text { for every } i \in\{0,1, \ldots, N-1\},
$$

where $A_{i} \in \mathbb{R}^{d \times d}$ invertible and $t_{i} \in \mathbb{R}^{d}$.

The fractal curve generated from $\mathcal{S}$ is the unique non-empty compact set $\Gamma$, for which

$$
\Gamma=\bigcup_{i=0}^{N-1} f_{i}(\Gamma) .
$$

If $\mathcal{S}$ is an affine zipper then we call $\Gamma$ a self-affine curve.

For an illustration see Figure 1. It shows the first (red), second (green) and third (black) level cylinders of the image of $[0,1]^{2}$. The cross-condition ensures that $\Gamma$ is a continuous curve.

The dimension theory of self-affine curves is far from being well understood. The Hausdorff dimension of such curves is known only in a very few cases. The usual

Date: 21st February 2018.

2010 Mathematics Subject Classification. Primary 28A80 Secondary 26A27 26A30

Key words and phrases. affine zippers, pointwise Hölder exponent, multifractal analysis, pressure function, iterated function system, de Rham curve. 


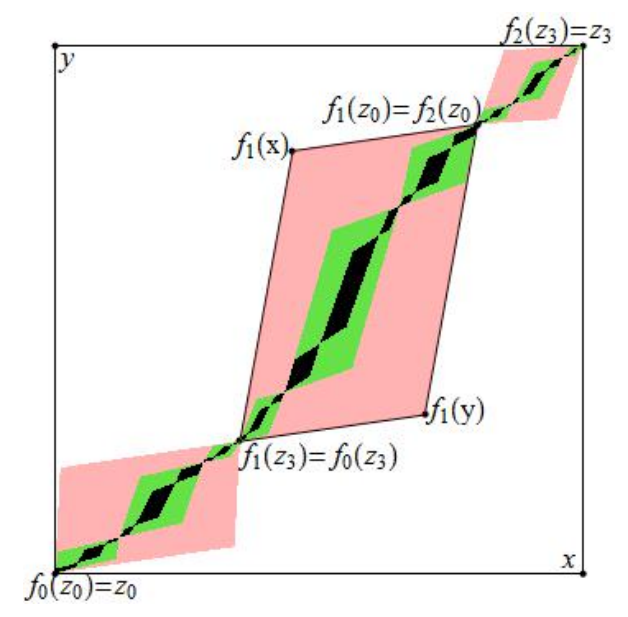

FiguRE 1. An affine zipper with $N=3$ maps and signature $\varepsilon=(0,1,0)$.

techniques, like self-affine transversality, see Falconer [14], Jordan, Pollicott and Simon [26], destroys the curve structure. Ledrappier [28] gave a sufficient condition to calculate the Hausdorff dimension of some fractal curves, and Solomyak [40] applied it to calculate the dimension of the graph of the Takagi function for typical parameters. Feng and Käenmäki [18] characterized self-affine systems, which have analytic curve attractor. Let us denote the $s$-dimensional Hausdorff measure and the Hausdorff dimension of a set $A$ by $\mathcal{H}^{s}(A)$ and $\operatorname{dim}_{H} A$, respectively. Moreover, let us denote the Packing and (upper) box-counting dimension of a set $A$ by $\operatorname{dim}_{P} A$ and $\operatorname{dim}_{B} A$, respectively. For basic properties and definition of Hausdorff-, Packing- and box-counting dimension, we refer to [15].

Bandt and Kravchenko [2] studied some smoothness properties of self-affine curves, especially the tangent lines of planar self-affine curves. The main purpose of this paper is to analyse the pointwise regularity of affine curves under some parametrization. Let us recall the definition of pointwise Hölder exponent of a real valued function $g$, see for example [24, eq. (1.1)]. We say that $g \in C^{\beta}(x)$ if there exist a $\delta>0, C>0$ and a polynomial $P$ with degree at most $\lfloor\beta\rfloor$ such that

$$
|g(y)-P(y-x)| \leq C|x-y|^{\beta} \text { for every } y \in B_{\delta}(x),
$$

where $B_{\delta}(x)$ denotes the ball with radius $\delta$ centered at $x$. Let $\alpha_{p}(x)=\sup \{\beta: g \in$ $\left.C^{\beta}(x)\right\}$. We call $\alpha_{p}(x)$ the pointwise Hölder exponent of $g$ at the point $x$.

We call $F: \mathbb{R}^{m} \mapsto \mathbb{R}$ a self-similar function if there exists a bounded open set $U \subset \mathbb{R}^{m}$, and contracting similarities $g_{1}, \ldots, g_{k}$ of $\mathbb{R}^{m}$ such that $g_{i}(U) \cap g_{j}(U)=\emptyset$ and $g_{i}(U) \subset U$ for every $i \neq j$, and a smooth function $g: \mathbb{R}^{m} \mapsto \mathbb{R}$, and real numbers $\left|\lambda_{i}\right|<1$ for $i=1, \ldots, k$ such that

$$
F(x)=\sum_{i=1}^{k} \lambda_{i} F\left(g_{i}^{-1}(x)\right)+g(x),
$$

see [25, Definition 2.1]. The multifractal formalism of the pointwise Hölder exponent of self-similar functions was studied in several aspects, see for example Aouidi and Slimane [1], Slimane [6, 7, 5] and Saka [38].

Hutchinson [22] showed that the family of contracting functions $g_{1}, \ldots, g_{k}$ has a unique, non-empty compact invariant set $\Omega$ (called the attractor of $\Phi=\left\{g_{1}, \ldots, g_{k}\right\}$ ), 
i.e. $\Omega=\bigcup_{i=1}^{k} g_{i}(\Omega)$. We note that in the case of self-similar function, the graph of $F$ (denoted by $\operatorname{Graph}(F)$ ) over the set $\Omega$ can be written as the unique, non-empty, compact invariant set of the family of functions $S_{1}, \ldots, S_{k}$ in $\mathbb{R}^{m+1}$, where

$$
S_{i}(x, y)=\left(g_{i}(x), \lambda_{i} y+g\left(g_{i}(x)\right)\right) .
$$

In this paper, we study the local regularity of a generalized version of selfsimilar functions. Namely, let $\boldsymbol{\lambda}=\left(\lambda_{0}, \ldots, \lambda_{N-1}\right)$ be a probability vector. Let us subdivide the interval $[0,1]$ according to the probability vector $\boldsymbol{\lambda}$ and signature $\varepsilon=\left(\varepsilon_{0}, \ldots, \varepsilon_{N-1}\right), \varepsilon_{i} \in\{0,1\}$ of the zipper $\mathcal{S}$. Let $g_{i}$ be the affine function mapping the unit interval $[0,1]$ to the $i$ th subinterval of the division which is order-preserving or order-reversing according to the signature $\varepsilon_{i}$. That is, the interval $[0,1]$ is the attractor of the iterated function system

$$
\Phi=\left\{g_{i}: x \mapsto(-1)^{\varepsilon_{i}} \lambda_{i} x+\gamma_{i}\right\}_{i=0}^{N-1},
$$

where $\gamma_{i}=\sum_{j=0}^{i-1} \lambda_{j}+\varepsilon_{i} \lambda_{i}$. Let $\Psi=\left\{S_{i}\right\}_{i=0}^{N-1}$ be an IFS on $\mathbb{R}^{d+1}$ such that

$$
S_{i}(x, y)=\left(g_{i}(x), A_{i} y+t_{i}\right)
$$

It is easy to see that if $\Lambda$ is the attractor of $\Psi$ then for every $x \in[0,1]$ there exists a unique $y \in \mathbb{R}^{d}$ such that $(x, y) \in \Lambda$. Thus, we can define a function $v:[0,1] \mapsto \Gamma \subset \mathbb{R}^{d}$ such that $v(x)=y$ if $(x, y) \in \Lambda$. The function $v$ satisfies the functional equation

$$
v(x)=f_{i}\left(v\left(g_{i}^{-1}(x)\right)\right) \text { if } x \in g_{i}([0,1]) .
$$

We note that $g_{i}^{-1}(x)=\frac{x-\gamma_{i}}{(-1)^{\varepsilon_{i}} \lambda_{i}}$, and $g_{i}^{-1}(x) \in[0,1]$ if and only if $x \in g_{i}([0,1])$. Moreover, if $\left\{f_{i}\right\}_{i=0}^{N-1}$ is a self-affine zipper then $v$ is continuous.

We call $v$ as the linear parametrization of $\Gamma$. Such linear parameterizations occur in the study of Wavelet functions in a natural way, see for example Protasov [36], Protasov and Guglielmi [37], and Seuret [39]. A particular example for (1.3) is the de Rham's curve, see Section 5 for details including an example of a graph of $v$ generated by the de Rham's curve.

The main difference between the self-similar function $F$ defined in (1.1) and $v$ defined in (1.3) is the contraction part. Namely, while $F$ is a real valued function rescaled by only a real number, the function $v$ is $\mathbb{R}^{d}$ valued and a strict affine transformation is acting on it. This makes the study of such functions more difficult. As a slight abuse of the appellation of the pointwise Hölder exponent, we use another exponent $\alpha(x)$ of the function $v$ at a point $x \in[0,1]$

$$
\alpha(x)=\liminf _{y \rightarrow x} \frac{\log \|v(x)-v(y)\|}{\log |x-y|} .
$$

We note that if $\alpha_{p}(x)<1$ or $\alpha(x)<1$ then $\alpha_{p}(x)=\alpha(x)$. Otherwise, we have only $\alpha(x) \leq \alpha_{p}(x)$.

When the liminf in (1.4) exists as a limit, then we say that $v$ has a regular pointwise Hölder exponent $\alpha_{r}(x)$ at a point $x \in[0,1]$, i.e.

$$
\alpha_{r}(x)=\lim _{y \rightarrow x} \frac{\log \|v(x)-v(y)\|}{\log |x-y|} .
$$


Let us define the level sets of the (regular) pointwise Hölder exponent by

$$
\begin{aligned}
E(\beta) & =\{x \in[0,1]: \alpha(x)=\beta\} \text { and } \\
E_{r}(\beta) & =\left\{x \in[0,1]: \alpha_{r}(x)=\beta\right\} .
\end{aligned}
$$

Our goal is to perform multifractal analysis, i.e. to study the possible values, which occur as (regular) pointwise Hölder exponents, and determine the magnitude of the sets, where it appears. This property was studied for several types of singular functions, for example for wavelets by Barral and Seuret [4], Seuret [39], for Weierstrass-type functions Otani [33], for complex analogues of the Takagi function by Jaerisch and Sumi [23] or for different functional equations by Coiffard, Melot and Willer [10], by Okamura [32] and by Slimane [7] etc.

The main difficulty in our approach is to handle the distance $\|v(x)-v(y)\|$. In the previous examples, the function $F$ defined with the equation (1.1) was scaled only by a constant. Roughly speaking

$$
\left\|F\left(g_{i_{1}, \ldots, i_{n}}(x)\right)-F\left(g_{i_{1}, \ldots, i_{n}}(y)\right)\right\| \approx\left|\lambda_{i_{1}} \cdots \lambda_{i_{n}}\right|\|F(x)-F(y)\| .
$$

In the case of self-affine systems, this is not true anymore. That is,

$$
\left\|v\left(g_{i_{1}, \ldots, i_{n}}(x)\right)-v\left(g_{i_{1}, \ldots, i_{n}}(y)\right)\right\| \approx\left\|A_{i_{1}} \cdots A_{i_{n}}(v(x)-v(y))\right\| .
$$

However, in general $\left\|A_{i_{1}} \cdots A_{i_{n}}(v(x)-v(y))\right\| \not \approx\left\|A_{i_{1}} \cdots A_{i_{n}}\right\|\|v(x)-v(y)\|$. In order to be able to compare the distance $\left\|v\left(g_{i_{1}, \ldots, i_{n}}(x)\right)-v\left(g_{i_{1}, \ldots, i_{n}}(y)\right)\right\|$ with the norm of the product of matrices, we need an extra assumption on the family of matrices.

Let us denote by $M^{o}$ the interior and by $\bar{M}$ the closure of a set $M \subseteq \mathbb{P R}^{d-1}$. For a point $v \in \mathbb{R}^{d}$, denote $\langle v\rangle$ equivalence class of $v$ in the projective space $\mathbb{P R}^{d-1}$. Every invertible matrix $A$ defines a natural map on the projective space $\mathbb{P R}^{d-1}$ by $\langle v\rangle \mapsto\langle A v\rangle$. As a slight abuse of notation, we denote this function by $A$ too.

Definition 1.2. We say that a family of matrices $\mathcal{A}=\left\{A_{0}, \ldots, A_{N-1}\right\}$ have dominated splitting of index-1 if there exists a non-empty open subset $M \subset \mathbb{P R}^{d-1}$ with a finite number of connected components with pairwise disjoint closure such that

$$
\bigcup_{i=0}^{N-1} A_{i} \bar{M} \subset M^{o},
$$

and there is a $d-1$ dimensional hyperplane that is transverse to all elements of $\bar{M}$. We call the set $M$ a multicone.

We adapted the definition of dominated splitting of index- 1 from the paper of Bochi and Gourmelon [8]. They showed that the tuple of matrices $\mathcal{A}$ satisfies the property in Definition 1.2 if and only if there exist constants $C>0$ and $0<\tau<1$ such that

$$
\frac{\alpha_{2}\left(A_{i_{1}} \cdots A_{i_{n}}\right)}{\alpha_{1}\left(A_{i_{1}} \cdots A_{i_{n}}\right)} \leq C \tau^{n}
$$

for every $n \geq 1$ and $i_{0}, \ldots, i_{n-1} \in\{0, \ldots, N-1\}$, where $\alpha_{i}(A)$ denotes the $i$ th largest singular value of the matrix $A$. That is, the weakest contracting direction and the stronger contracting directions are strongly separated away (splitted), $\alpha_{1}$ dominates $\alpha_{2}$. This condition makes it easier to handle the growth rate of the norm of matrix products, which will be essential in our later studies.

We note that for example a tuple $\mathcal{A}$ formed by matrices with strictly positive elements, satisfies the dominated splitting of index- 1 of $M=\left\langle\left\{x \in \mathbb{R}^{d}: x_{i}>0, i=\right.\right.$ 
$1, \ldots, d\}\rangle$. Throughout the paper we work with affine zippers, where we assume that the matrices $A_{i}$ have dominated splitting of index-1. For more details, see Section 2 and [8].

For a subset $M$ of $\mathbb{P R}^{d-1}$ and a point $x \in \mathbb{R}^{d}$, let

$$
M(x)=\left\{y \in \mathbb{R}^{d}:\langle y-x\rangle \in M\right\} .
$$

We call the set $M(x)$ a cone centered at $x$.

We say that $\mathcal{S}$ satisfies the strong open set condition (SOSC), if there exists an open bounded set $U$ such that $f_{i}(U) \subseteq U$,

$$
f_{i}(U) \cap f_{j}(U)=\emptyset \text { for every } i \neq j \text { and } \Gamma \cap U \neq \emptyset .
$$

We call $\mathcal{S}$ a non-degenerate system, if it satisfies the SOSC and $\left\langle z_{N}-z_{0}\right\rangle \notin$ $\bigcap_{k=0}^{\infty} \bigcap_{|\overline{\mid}|=k} A_{\bar{\imath}}^{-1}\left(M^{c}\right)$, where for a finite length word $\bar{\imath}=i_{1} \ldots i_{k}, A_{\bar{\imath}}$ denotes the matrix product $A_{i_{1}} A_{i_{2}} \ldots A_{i_{k}}$. We note that the non-degenerate condition guarantees that the curve $v:[0,1] \mapsto \mathbb{R}^{d}$ is not self-intersecting and it is not contained in a strict hyperplane of $\mathbb{R}^{d}$.

Denote by $P(t)$ the pressure function which is defined as the unique root of the equation

$$
0=\lim _{n \rightarrow \infty} \frac{1}{n} \log \sum_{i_{1}, \ldots, i_{n}=0}^{N-1}\left\|A_{i_{1}} \cdots A_{i_{n}}\right\|^{t}\left(\lambda_{i_{1}} \cdots \lambda_{i_{n}}\right)^{-P(t)} .
$$

A considerable attention has been paid for pressures, which are defined by matrix norms, see for example Käenmäki [27], Feng and Shmerkin [20], and Morris $[29,30]$. Feng [16] and later Feng and Lau [19] studied the properties of the pressure $P$ for positive and non-negative matrices. In Section 2, we extend these results for the dominated splitting of index-1 case. Namely, we will show that the function $P: \mathbb{R} \mapsto \mathbb{R}$ is continuous, concave, monotone increasing, and continuously differentiable.

Unfortunately, even for positive matrices, the computation of the precise values of $P(t)$ is hopeless. For a fast approximation algorithm, see Pollicott and Vytnova [34].

Let $d_{0}>0$ be the unique real number such that

$$
0=\lim _{n \rightarrow \infty} \frac{1}{n} \log \sum_{|\bar{\imath}|=n}\left\|A_{\bar{\imath}}\right\|^{d_{0}} .
$$

Observe that for every $n \geq 1,\left\{f_{\bar{\imath}}(\bar{U}):|\bar{\imath}|=n\right\}$ defines a cover of $\Gamma$. But since $\Gamma$ is a curve and thus $\operatorname{dim}_{H} \Gamma \geq 1$, and since every $f_{\bar{\imath}}(\bar{U})$ can be covered by a ball with radius $\left\|A_{\bar{\imath}}\right\||U|, d_{0} \geq 1$.

Let

$$
\alpha_{\min }=\lim _{t \rightarrow+\infty} \frac{P(t)}{t}, \alpha_{\max }=\lim _{t \rightarrow-\infty} \frac{P(t)}{t} \text { and } \widehat{\alpha}=P^{\prime}(0) .
$$

The values $\alpha_{\min }$ and $\alpha_{\max }$ correspond to the logarithm of the joint- and the lower-spectral radius defined by Protasov [36].

Now, we state our main theorems on the pointwise Hölder exponents.

Theorem 1.3. Let $v$ be a linear parametrization of $\Gamma$ defined by a non-degenerate system $\mathcal{S}$. Then there exists a constant $\widehat{\alpha}$, defined in (1.8), such that for $\mathcal{L}$-a.e. 
$x \in[0,1], \alpha(x)=\widehat{\alpha} \geq 1 / d_{0}$. In addition, there exists an $\varepsilon>0$ such that for every $\beta \in[\widehat{\alpha}, \widehat{\alpha}+\varepsilon]$

$$
\operatorname{dim}_{H}\{x \in[0,1]: \alpha(x)=\beta\}=\inf _{t \in \mathbb{R}}\{t \beta-P(t)\} .
$$

Moreover, (1.9) can be extended for every $\beta \in\left[\alpha_{\min }, \widehat{\alpha}+\varepsilon\right]$ if $v$ satisfies

$$
\lambda_{0}=\lambda_{N-1} \text { and } \lim _{k \rightarrow \infty} \frac{\left\|A_{0}^{k}\right\|}{\left\|A_{N-1}^{k}\right\|}=1 .
$$

Furthermore, the functions $\beta \mapsto \operatorname{dim}_{H} E(\beta)$ and $\beta \mapsto \operatorname{dim}_{H} E_{r}(\beta)$ are continuous and concave on their respective domains.

In the following, we give a sufficient condition to extend the previous result, where (1.9) holds to the complete spectrum $\left[\alpha_{\min }, \alpha_{\max }\right]$. As a slight abuse of notation for every $\theta \in \mathbb{P R}^{d-1}$, we say that $\underline{0} \neq v \in \theta$ if $\langle v\rangle=\theta$.

Assumption A. For a non-degenerate affine zipper $\mathcal{S}=\left\{f_{i}: x \mapsto A_{i} x+t_{i}\right\}_{i=0}^{N-1}$ with vertices $\left\{z_{0}, \ldots, z_{N}\right\}$ assume that there exists a convex, simply connected closed cone $C \subset \mathbb{P R}^{d-1}$ such that

(1) $\bigcup_{i=1}^{N} A_{i} C \subset C^{o}$ and for every $\underline{0} \neq v \in \theta \in C,\left\langle A_{i} v, v\right\rangle>0$,

(2) $\left\langle z_{N}-z_{0}\right\rangle \in C^{o}$.

Observe that if $\mathcal{S}$ satisfies Assumption A then it satisfies the strong open set condition with respect to the set $U$, which is the bounded component of $C^{o}\left(z_{0}\right) \cap$ $C^{o}\left(z_{N}\right)$. We note that if all the matrices have strictly positive elements and the zipper has signature $(0, \ldots, 0)$ then Assumption A holds.

Theorem 1.4. Let $\mathcal{S}$ be an affine zipper satisfying Assumption A. Then for every $\beta \in\left[\widehat{\alpha}, \alpha_{\max }\right]$

$$
\operatorname{dim}_{H}\{x \in[0,1]: \alpha(x)=\beta\}=\inf _{t \in \mathbb{R}}\{t \beta-P(t)\},
$$

and for every $\beta \in\left[\alpha_{\min }, \alpha_{\max }\right]$

$$
\operatorname{dim}_{H}\left\{x \in[0,1]: \alpha_{r}(x)=\beta\right\}=\inf _{t \in \mathbb{R}}\{t \beta-P(t)\} .
$$

Moreover, if $\mathcal{S}$ satisfies (1.10) then (1.11) can be extended for every $\beta \in\left[\alpha_{\min }, \alpha_{\max }\right]$.

The functions $\beta \mapsto \operatorname{dim}_{H} E(\beta)$ and $\beta \mapsto \operatorname{dim}_{H} E_{r}(\beta)$ are continuous and concave on their respective domains.

Assumption A has another important role. In Theorem 1.4, we calculated the spectrum for the regular Hölder exponent, providing that it exists. We show that the existence of the regular Hölder exponent for Lebesgue typical points is equivalent to Assumption A.

Theorem 1.5. Let $\mathcal{S}$ be a non degenerate system. Then the regular Hölder exponent exists for Lebesgue almost every point if and only if $\mathcal{S}$ satisfies Assumption A. In particular, $\alpha_{r}(x)=P^{\prime}(0)$ for Lebesgue almost every $x \in[0,1]$.

Remark 1.6. In the sequel, to keep the notation tractable we assume the signature $\varepsilon=(0, \ldots, 0)$. The results carry over for general signatures, and the proofs can be easily modified for the general signature case, see Remark 5.3. 
The organization of the paper is as follows. In Section 2 we prove several properties of the pressure function $P(t)$, extending the works of $[16,19]$ to the dominated splitting of index-1 case using [8]. We prove Theorem 1.3 in Section 3. Section 4 contains the proofs of Theorems 1.4 and 1.5 when the zipper satisfies Assumption A. Finally, as an application in Section 5, we show that our results can be applied to de Rham's curve, giving finer results than existing ones in the literature.

\section{Pressure for matrices With Dominated Splitting of IndeX-1}

In this section, we generalize the result of Feng [16], and Feng and Lau [19]. In [19] the authors studied the pressure function and multifractal properties of Lyapunov exponents for products of positive matrices. Here, we extend their results for a more general class of matrices by using Bochi and Gourmelon [8] for later usage.

Let $\Sigma$ be the set of one side infinite length words of symbols $\{0, \ldots, N-1\}$, i.e. $\Sigma=\{0, \ldots, N-1\}^{\mathbb{N}}$. Let $\sigma$ denote the left shift on $\Sigma$, its $n$-fold composition by $\sigma^{n} \mathbf{i}=\left(i_{n+1}, i_{n+2}, \ldots\right)$. We use the standard notation $\mathbf{i} \mid n$ for $i_{1}, \ldots, i_{n}$ and

$$
\left[\left.\mathbf{i}\right|_{n}\right]:=\left\{\mathbf{j} \in \Sigma: j_{1}=i_{1}, \ldots, j_{n}=i_{n}\right\} .
$$

Let us denote the set of finite length words by $\Sigma^{*}=\bigcup_{n=0}^{\infty}\{0, \ldots, N-1\}^{n}$, and for an $\bar{\imath} \in \Sigma^{*}$, let us denote the length of $\bar{\imath}$ by $|\bar{\imath}|$. For a finite word $\bar{\imath} \in \Sigma^{*}$ and for a $\mathbf{j} \in \Sigma$, denote $\bar{\imath} \mathbf{j}$ the concatenation of the finite word $\bar{\imath}$ with $\mathbf{j}$.

Denote $\mathbf{i} \wedge \mathbf{j}$ the length of the longest common prefix of $\mathbf{i}, \mathbf{j} \in \Sigma$, i.e. $\mathbf{i} \wedge \mathbf{j}=$ $\min \left\{n-1: i_{n} \neq j_{n}\right\}$. Let $\boldsymbol{\lambda}=\left(\lambda_{0}, \ldots, \lambda_{N-1}\right)$ be a probability vector and let $d(\mathbf{i}, \mathbf{j})$ be the distance on $\Sigma$ with respect to $\lambda$. Namely,

$$
d(\mathbf{i}, \mathbf{j})=\prod_{n=1}^{\mathbf{i} \wedge \mathbf{j}} \lambda_{i_{n}}=: \lambda_{\left.\mathbf{i}\right|_{\mathbf{i} \wedge \mathbf{j}}} .
$$

If $\mathbf{i} \wedge \mathbf{j}=0$ then by definition $\left.\mathbf{i}\right|_{\mathbf{i} \wedge \mathbf{j}}=\emptyset$ and $\lambda_{\emptyset}=1$. In the sequel, whenever we use Hausdorff dimension in $\Sigma$ it is with respect to this metric $d(\mathbf{i}, \mathbf{j})$. For every $r>0$, we define a partition $\Xi_{r}$ of $\Sigma$ by

$$
\Xi_{r}=\left\{\left[i_{1}, \ldots, i_{n}\right]: \lambda_{i_{1}} \cdots \lambda_{i_{n}} \leq r<\lambda_{i_{1}} \cdots \lambda_{i_{n-1}}\right\} .
$$

For a matrix $A$ and a subspace $\theta$, denote $\|A \mid \theta\|$ the norm of $A$ restricted to $\theta$, i.e. $\|A \mid \theta\|=\sup _{v \in \theta}\|A v\| /\|v\|$. In particular, if $\theta$ has dimension one $\|A \mid \theta\|=\|A v\| /\|v\|$ for any $\underline{0} \neq v \in \theta$. Denote $G(d, k)$ the Grassmanian manifold of $k$ dimensional subspaces of $\mathbb{R}^{d}$. We define the angle between a 1 dimensional subspace $E$ and a $d-1$ dimensional subspace $F$ as usual, i.e.

$$
\varangle(E, F)=\arccos \left(\frac{\left\langle v, \operatorname{proj}_{F} v\right\rangle}{\left\|\operatorname{proj}_{F} v\right\|\|v\|}\right),
$$

where $\underline{0} \neq v \in E$ arbitrary and $\operatorname{proj}_{F}$ denotes the orthogonal projection onto $F$.

The following theorem collects the most relevant properties of a family of matrices with dominated splitting of index-1.

Theorem 2.1. [8, Theorem A, Theorem B, Claim on p. 228] Suppose that a finite set of matrices $\left\{A_{0}, \ldots, A_{N-1}\right\}$ satisfies the dominated splitting of index-1 with multicone $M$. Then there exist Hölder continuous functions $E: \Sigma \mapsto \mathbb{P}^{d-1}$ and $F: \Sigma \mapsto G(d, d-1)$ such that

(1) $E(\mathbf{i})=A_{i_{1}} E(\sigma \mathbf{i})$ for every $\mathbf{i} \in \Sigma$, 
(2) $F(\mathbf{i})=A_{i_{1}}^{-1} F(\sigma \mathbf{i})$ for every $\mathbf{i} \in \Sigma$,

(3) there exists $\beta>0$ such that $\varangle(E(\mathbf{i}), F(\mathbf{j}))>\beta$ for every $\mathbf{i}, \mathbf{j} \in \Sigma$,

(4) there exist constants $C \geq 1$ and $0<\tau<1$ such that

$$
\frac{\alpha_{2}\left(A_{\left.\mathbf{i}\right|_{n}}\right)}{\left\|A_{\left.\mathbf{i}\right|_{n}}\right\|} \leq C \tau^{n}
$$

for every $\mathbf{i} \in \Sigma$ and $n \geq 1$,

(5) there exists a constant $C>0$ such that $\left\|A_{\left.\mathbf{i}\right|_{n}} \mid E\left(\sigma^{n} \mathbf{i}\right)\right\| \geq C\left\|A_{\left.\mathbf{i}\right|_{n}}\right\|$ for every $\mathbf{i} \in \Sigma$,

(6) there exists a constant $C>0$ such that $\left\|A_{\left.\mathbf{i}\right|_{n}} \mid F\left(i_{n} \ldots i_{1} \mathbf{j}\right)\right\| \leq C \alpha_{2}\left(A_{\left.\mathbf{i}\right|_{n}}\right)$ for every $\mathbf{i}, \mathbf{j} \in \Sigma$.

There are a few simple consequences of Theorem 2.1. First, if $M$ is the multicone from Definition 1.2, then by Theorem 2.1(1)

$$
E(\mathbf{i})=\bigcap_{n=1}^{\infty} A_{i_{1}} \cdots A_{i_{n}}(M)
$$

and for every $V \in M, A_{i_{1}} \cdots A_{i_{n}} V \rightarrow E(\mathbf{i})$ uniformly (independently of $V$ ). Hence, by property (5), there exists a constant $C^{\prime}>0$ such that for every $V \in M$ and every $\bar{\imath} \in \Sigma^{*}$,

$$
\left\|A_{\bar{\imath}} \mid V\right\| \geq C^{\prime}\left\|A_{\bar{\imath}}\right\|
$$

So, this gives us a strong control over the growth rate of matrix products on subspaces in $M$.

Remark 2.2. We note if the multicone $M$ in Definition 1.2 has only one connected component then it can be chosen to be simply connected and convex. Indeed, since $\bar{M}$ is separated away from the strong stable subspaces $F$ then $\mathrm{cv}(\bar{M})$ must be separated away from every $d-1$ dimensional strong stable subspace, as well, where $\operatorname{cv}(\bar{M})$ denotes the convex hull of $\bar{M}$. Thus $A_{i}(\operatorname{cv}(\bar{M})) \subset \operatorname{cv}(\bar{M})^{o}$ for every $i$.

Second, property (1) of Theorem 2.1 also implies that

$$
\left\|A_{\left.\mathbf{i}\right|_{n}}\left|E\left(\sigma^{n} \mathbf{i}\right)\left\|=\prod_{k=1}^{n}\right\| A_{i_{k}}\right| E\left(\sigma^{k} \mathbf{i}\right)\right\| .
$$

Indeed, since $E(\mathbf{i})$ is a one dimensional subspace, for every $v \in E\left(\sigma^{n} \mathbf{i}\right)$

$$
\left\|A_{\mathbf{i} \mid n}\left|E\left(\sigma^{n} \mathbf{i}\right)\left\|=\frac{\left\|A_{\mathbf{i} \mid n} v\right\|}{\|v\|}=\prod_{k=1}^{n} \frac{\left\|A_{i_{k} \ldots i_{n}} v\right\|}{\left\|A_{i_{k+1}, \ldots, i_{n}} v\right\|}=\prod_{k=1}^{n}\right\| A_{i_{k}}\right| E\left(\sigma^{k} \mathbf{i}\right)\right\| .
$$

Moreover, since $E(\mathbf{i})$ is Hölder-continuous, the function $\psi(\mathbf{i}):=\log \left\|A_{i_{1}} \mid E(\sigma \mathbf{i})\right\|$ is also Hölder-continuous. That is, there exist $C>0$ and $0<\tau<1$ such that

$$
|\psi(\mathbf{i})-\psi(\mathbf{j})| \leq C \tau^{\mathbf{i} \wedge \mathbf{j}} .
$$

It is easy to see that (2.5) holds if and only if $\psi$ is Hölder-continuous with respect to the metric $d$ defined in (2.1). by

Finally, (2.5) implies that for every $t$, the potential function $\varphi_{t}: \Sigma \mapsto \mathbb{R}$ defined

$$
\varphi_{t}(\mathbf{i}):=\log \left(\left\|A_{i_{1}} \mid E(\sigma \mathbf{i})\right\|^{t} \lambda_{i_{1}}^{-P(t)}\right)=t \psi(\mathbf{i})-P(t) \log \lambda_{i_{1}}
$$

is Hölder-continuous w.r.t the metric $d$, where $P(t)$ was defined in (1.6). Thus, by [9, Theorem 1.4], for every $t \in \mathbb{R}$ there exists a unique $\sigma$-invariant, ergodic 
probability measure $\mu_{t}$ on $\Sigma$ such that there exists a constant $C(t)>1$ such that for every $\mathbf{i} \in \Sigma$ and every $n \geq 1$

$$
C(t)^{-1} \leq \frac{\mu_{t}\left(\left[\left.\mathbf{i}\right|_{n}\right]\right)}{\prod_{k=0}^{n-1} e^{\varphi_{t}\left(\sigma^{k^{k}}\right)}}=\frac{\mu_{t}\left(\left[\left.\mathbf{i}\right|_{n}\right]\right)}{\left\|A_{\left.\mathbf{i}\right|_{n}} \mid E\left(\sigma^{n} \mathbf{i}\right)\right\|^{t} \cdot \lambda_{\left.\mathbf{i}\right|_{n}}^{-P(t)}} \leq C(t),
$$

where the equality follows from substituting (2.6) and (2.4).

Moreover, for the Hausdorff dimension w.r.t. the metric $d$ defined in (2.1)

$$
\operatorname{dim}_{H} \mu_{t}=\frac{h_{\mu_{t}}}{\chi_{\mu_{t}}}
$$

where

$$
\begin{aligned}
& h_{\mu_{t}}=\lim _{n \rightarrow \infty} \frac{-1}{n} \sum_{|\bar{\imath}|=n} \mu_{t}([\bar{\imath}]) \log \mu_{t}([\bar{l}])=-\int \varphi_{t}(\mathbf{i}) d \mu_{t}(\mathbf{i}), \\
& \chi_{\mu_{t}}=\lim _{n \rightarrow \infty} \frac{-1}{n} \sum_{|\bar{\imath}|=n} \mu_{t}([\bar{\imath}]) \log \lambda_{\bar{\imath}}=-\int \log \lambda_{i_{1}} d \mu_{t}(\mathbf{i}) .
\end{aligned}
$$

We call $\chi_{\mu_{t}}$ the Lyapunov exponent of $\mu_{t}$ and $h_{\mu_{t}}$ the entropy of $\mu_{t}$.

Lemma 2.3. The map $t \mapsto P(t)$ is continuous, concave, monotone increasing on $\mathbb{R}$.

Proof. Since $\mu_{t}$ is a probability measure on $\Sigma$ and $\Xi_{r}$ is a partition we get

$$
0=\frac{\log \sum_{\bar{\imath} \in \Xi_{r}} \mu_{t}([\bar{\imath}])}{\log r} \text { for every } r>0
$$

and by (2.7), Theorem 2.1(5) and (1.6)

$$
P(t)=\lim _{r \rightarrow 0+} \frac{\log \sum_{\bar{\imath} \in \Xi_{r}}\left\|A_{\bar{\imath}}\right\|^{t}}{\log r} .
$$

Using this form it can be easily seen that $t \mapsto P(t)$ is continuous, concave and monotone increasing.

By Lemma 2.3, the potential $\varphi_{t}$ depends continuously on $t$. Moreover, by (2.5), $\left|\varphi_{t}(\mathbf{i})-\varphi_{t}(\mathbf{j})\right| \leq C t \tau^{\mathbf{i} \wedge \mathbf{j}}$. Thus, the Perron-Frobenius operator

$$
\left(T_{t}(g)\right)(\mathbf{i})=\sum_{i=0}^{N-1} e^{\varphi_{t}(i \mathbf{i})} g(i \mathbf{i})
$$

depends continuously on $t$. Hence, both the unique eigenfunction $h_{t}$ of $T_{t}$ and the eigenmeasure $\nu_{t}$ of the dual operator $T_{t}^{*}$ depend continuously on $t$. Since $d \mu_{t}=h_{t} d \nu_{t}$, see [9, Theorem 1.16], we got that $t \mapsto \mu_{t}$ is continuous in weak*topology. Hence, by (2.9) and (2.10), $t \mapsto h_{\mu_{t}}$ and $t \mapsto \chi_{\mu_{t}}$ are continuous on $\mathbb{R}$.

Proposition 2.4. The map $t \mapsto P(t)$ is continuously differentiable on $\mathbb{R}$. Moreover, for every $t \in \mathbb{R}$

$$
\operatorname{dim}_{H} \mu_{t}=t P^{\prime}(t)-P(t)
$$

and

$$
\lim _{n \rightarrow \infty} \frac{\log \left\|A_{i_{1}} \cdots A_{i_{n}}\right\|}{\log \lambda_{i_{1}} \cdots \lambda_{i_{n}}}=P^{\prime}(t) \text { for } \mu_{t} \text {-almost every } \mathbf{i} \in \Sigma \text {. }
$$


Proof. We recall [21, Theorem 3.1]. That is, since $\mu_{t}$ is a Gibbs measure

$$
\tau_{\mu_{t}}(q)=\lim _{r \rightarrow 0+} \frac{\log \sum_{\bar{i} \in \Xi_{r}} \mu_{t}([\bar{\imath}])^{q}}{\log r}
$$

is differentiable at $q=1$ and $\tau_{\mu_{t}}^{\prime}(1)=\operatorname{dim}_{H} \mu_{t}$. On the other hand, by (2.7) and (2.11)

$$
\tau_{\mu_{t}}(q)=P(t q)-P(t) q .
$$

Hence, by taking the derivative at $q=1$ we get that $P(t)$ is differentiable for every $t \in \mathbb{R} \backslash\{0\}$ and

$$
\operatorname{dim}_{H} \mu_{t}=t P^{\prime}(t)-P(t) .
$$

Let us observe that by (2.6), (2.8) and (2.9)

$$
\operatorname{dim}_{H} \mu_{t}=t \frac{-\int \log \left\|A_{i_{1}} \mid E(\sigma \mathbf{i})\right\| d \mu_{t}(\mathbf{i})}{-\int \log \lambda_{i_{1}} d \mu_{t}(\mathbf{i})}-P(t) .
$$

Thus,

$$
P^{\prime}(t)=\frac{-\int \log \left\|A_{i_{1}} \mid E(\sigma \mathbf{i})\right\| d \mu_{t}(\mathbf{i})}{-\int \log \lambda_{i_{1}} d \mu_{t}(\mathbf{i})} \text { for every } t \neq 0 .
$$

Since $t \mapsto \mu_{t}$ is continuous in weak*-topology we get that $t \mapsto P^{\prime}(t)$ is continuous on $\mathbb{R} \backslash\{0\}$. On the other hand, the left and right hand side limits of $P^{\prime}(t)$ at $t=0$ exist and are equal. Thus, $t \mapsto P(t)$ is continuously differentiable on $\mathbb{R}$.

By Theorem 2.1 (5), equation (2.4) and ergodicity of $\mu_{t}$ we get the last assertion of the proposition.

Let us observe that by the definition of pressure function (1.6), $P(0)=-1$ and thus, $\mu_{0}$ corresponds to the Bernoulli measure on $\Sigma$ with probabilities $\left(\lambda_{0}, \ldots, \lambda_{N-1}\right)$. That is,

$$
\mu_{0}\left(\left[i_{1}, \ldots, i_{n}\right]\right)=\lambda_{i_{1}} \cdots \lambda_{i_{n}} .
$$

Lemma 2.5. For every finite set of matrices $\mathcal{A}$ with dominated splitting of index-1, $P^{\prime}(0) \geq 1 / d_{0}, P^{\prime}\left(d_{0}\right) \leq 1 / d_{0}$. Moreover, $P^{\prime}(0)>1 / d_{0}$ if and only if $P^{\prime}\left(d_{0}\right)<1 / d_{0}$ if and only if $\mu_{d_{0}} \neq \mu_{0}$.

Proof. By the definition of $P(t),(1.6), P\left(d_{0}\right)=0$, where $d_{0}$ is defined in (1.7). Together with $P(0)=-1$ and the concavity and differentiability of $P(t)$ (by Lemma 2.3 and Proposition 2.4), we get $P^{\prime}(0) \geq 1 / d_{0}, P^{\prime}\left(d_{0}\right) \leq 1 / d_{0}$. Moreover, $P^{\prime}(0)>$ $1 / d_{0}$ if and only if $P^{\prime}\left(d_{0}\right)<1 / d_{0}$.

On the other hand, by Proposition 2.4

$$
\operatorname{dim}_{H} \mu_{d_{0}}=d_{0} P^{\prime}\left(d_{0}\right)=\lim _{n \rightarrow \infty} \frac{\log \left\|A_{\left.\mathbf{i}\right|_{n}}\right\|^{d_{0}}}{\log \lambda_{\left.\mathbf{i}\right|_{n}}}=\frac{h_{\mu_{d_{0}}}}{\chi_{\mu_{d_{0}}}} \text { for } \mu_{d_{0}} \text {-a.e. } \mathbf{i},
$$

where in the last equation we used the definition of $\mu_{d_{0}}$, the entropy and the Lyapunov exponent. Since $\operatorname{dim}_{H} \mu_{0}=1$, if $P^{\prime}\left(d_{0}\right)<1 / d_{0}$ then $\mu_{0} \neq \mu_{d_{0}}$. Otherwise, by [9, Theorem 1.22], for every $\sigma$-invariant, ergodic measure $\nu$ on $\Sigma$,

$$
\frac{h_{\nu}}{-\int \log \lambda_{i_{0}} d \nu(\mathbf{i})} \leq 1 \text { and } \frac{h_{\nu}}{-\int \log \lambda_{i_{0}} d \nu(\mathbf{i})}=1 \text { if and only if } \nu=\mu_{0} .
$$

Therefore, if $P^{\prime}\left(d_{0}\right)=1 / d_{0}$ then $\frac{h_{\mu_{0}}}{\chi_{\mu_{d_{0}}}}=1$ and so $\mu_{d_{0}}=\mu_{0}$. 
Lemma 2.6. For every $\alpha \in\left[\alpha_{\min }, \alpha_{\max }\right]$

$$
\operatorname{dim}_{H}\left\{\mathbf{i} \in \Sigma: \liminf _{m \rightarrow \infty} \frac{\log \left\|A_{\left.\mathbf{i}\right|_{m}}\right\|}{\log \lambda_{\left.\mathbf{i}\right|_{m}}} \leq \alpha\right\} \leq \inf _{t \geq 0}\{t \alpha-P(t)\}
$$

and

$$
\operatorname{dim}_{H}\left\{\mathbf{i} \in \Sigma: \limsup _{m \rightarrow \infty} \frac{\log \left\|A_{\left.\mathbf{i}\right|_{m}}\right\|}{\log \lambda_{\left.\mathbf{i}\right|_{m}}} \geq \alpha\right\} \leq \inf _{t \leq 0}\{t \alpha-P(t)\}
$$

Proof. For simplicity, we use the notations

$\underline{G}_{\alpha}=\left\{\mathbf{i} \in \Sigma: \liminf _{m \rightarrow \infty} \frac{\log \left\|A_{\left.\mathbf{i}\right|_{m}}\right\|}{\log \lambda_{\left.\mathbf{i}\right|_{m}}} \leq \alpha\right\}$ and $\bar{G}_{\alpha}=\left\{\mathbf{i} \in \Sigma: \limsup _{m \rightarrow \infty} \frac{\log \left\|A_{\left.\mathbf{i}\right|_{m}}\right\|}{\log \lambda_{\left.\mathbf{i}\right|_{m}}} \geq \alpha\right\}$.

Let $\varepsilon>0$ be arbitrary but fixed and let us define the following sets of cylinders:

$$
\underline{D}_{r}(\varepsilon)=\left\{[\bar{\imath}] \in \Xi_{\rho}: 0<\rho \leq r \text { and } \frac{\log \left\|A_{\bar{\imath}}\right\|}{\log \lambda_{\bar{\imath}}} \leq \alpha+\varepsilon\right\}
$$

and

$$
\bar{D}_{r}(\varepsilon)=\left\{[\bar{\imath}] \in \Xi_{\rho}: 0<\rho \leq r \text { and } \frac{\log \left\|A_{\bar{\imath}}\right\|}{\log \lambda_{\bar{\imath}}} \geq \alpha-\varepsilon\right\} .
$$

By definition, $\underline{D}_{r}(\varepsilon)$ is a cover of $\underline{G}_{\alpha}$ and respectively, $\bar{D}_{r}(\varepsilon)$ is a cover of $\bar{G}_{\alpha}$. Now let $\underline{C}_{r}(\varepsilon)$ and $\bar{C}_{r}(\varepsilon)$ be a disjoint set of cylinders such that

$$
\bigcup_{[\bar{l}] \in \underline{D}_{r}(\varepsilon)}[\bar{l}]=\bigcup_{[\bar{l}] \in \underline{C}_{r}(\varepsilon)}[\bar{\imath}] \text { and } \bigcup_{[\bar{\imath}] \in \bar{D}_{r}(\varepsilon)}[\bar{\imath}]=\bigcup_{[\bar{l}] \in \bar{C}_{r}(\varepsilon)}[\bar{l}] .
$$

Then by (2.7) and the definition of $\underline{C}_{r}(\varepsilon)$, for any $t \geq 0$

$$
\begin{aligned}
\mathcal{H}_{r}^{\alpha t-P(t)+(1+t) \varepsilon}\left(\underline{G}_{\alpha}\right) & \leq \sum_{[\bar{\imath}] \in \underline{C}_{r}(\varepsilon)} \lambda_{\bar{\imath}}^{(\alpha t-P(t)+(1+t) \varepsilon)} \\
& \leq \lambda_{\min }^{-1} r^{\varepsilon} \sum_{[\bar{\imath}] \in \underline{C}_{r}(\varepsilon)}\left\|A_{\bar{\imath} \|}\right\|^{t} \lambda_{\bar{\imath}}^{-P(t)} \\
& \leq C \lambda_{\min }^{-1} r^{\varepsilon} \sum_{[\bar{\imath}] \in \underline{C}_{r}(\varepsilon)} \mu_{t}([\overline{\bar{l}}]) \leq C \lambda_{\min }^{-1} r^{\varepsilon} .
\end{aligned}
$$

Hence, $\mathcal{H}^{\alpha t-P(t)+(1+t) \varepsilon}\left(\underline{G}_{\alpha}\right)=0$ for any $t>0$ and any $\varepsilon>0$, so (2.12) follows. The proof of (2.13) is similar by using the cover $\bar{C}_{r}(\varepsilon)$ of $\bar{G}_{\alpha}$.

We note that by the concavity of $P$

$$
\inf _{t \in \mathbb{R}}\{t \alpha-P(t)\}=\inf _{t \leq 0}\{t \alpha-P(t)\},
$$

for every and $\alpha \in\left[P^{\prime}(0), \alpha_{\max }\right]$,

$$
\inf _{t \in \mathbb{R}}\{t \alpha-P(t)\}=\inf _{t \geq 0}\{t \alpha-P(t)\},
$$

for every $\alpha \in\left[\alpha_{\min }, P^{\prime}(0)\right]$. 


\section{Pointwise Hölder exponent For NON-DEgenerate CuRves}

First, let us define the natural projections $\pi$ and $\Pi$ from the symbolic space $\Sigma$ to the unit interval $[0,1]$ and the curve $\Gamma$. We recall that we assumed that all the signatures of the affine zipper Definition 1.1 is 0 , and all the matrices are invertible. Therefore,

$$
\begin{aligned}
\pi(\mathbf{i}) & =\lim _{n \rightarrow \infty} g_{i_{1}} \circ \cdots \circ g_{i_{n}}(0)=\sum_{n=1}^{\infty} \lambda_{\left.\mathbf{i}\right|_{n-1}} \gamma_{i_{n}} \\
\Pi(\mathbf{i}) & =\lim _{n \rightarrow \infty} f_{i_{1}} \circ \cdots \circ f_{i_{n}}(\underline{0})=\sum_{n=1}^{\infty} A_{\left.\mathbf{i}\right|_{n-1}} t_{i_{n}} .
\end{aligned}
$$

Observe that by the definition of the linear parametrization $v$ of $\Gamma, v(\pi(\mathbf{i}))=\Pi(\mathbf{i})$.

In the analysis of the pointwise Hölder exponent $\alpha$, defined in (1.4), the points play important role which are far away symbolically but close on the self-affine curve. To be able to handle such points we introduce the following notation

$$
\mathbf{i} \vee \mathbf{j}= \begin{cases}\min \left\{\sigma^{\mathbf{i} \wedge \mathbf{j}+1} \mathbf{i} \wedge \mathbf{N}-\mathbf{1}, \sigma^{\mathbf{i} \wedge \mathbf{j}+1} \mathbf{j} \wedge \mathbf{0}\right\}, & \text { if } i_{\mathbf{i} \wedge \mathbf{j}+1}+1=j_{\mathbf{i} \wedge \mathbf{j}+1}, \\ \min \left\{\sigma^{\mathbf{i} \wedge \mathbf{j}+1} \mathbf{i} \wedge \mathbf{0}, \sigma^{\mathbf{i} \wedge \mathbf{j}+1} \mathbf{j} \wedge \mathbf{N}-\mathbf{1}\right\}, & \text { if } j_{\mathbf{i} \wedge \mathbf{j}+1}+1=i_{\mathbf{i} \wedge \mathbf{j}+1}, \\ 0, & \text { otherwise }\end{cases}
$$

where $\mathbf{0}$ denotes the $(0,0, \ldots)$ and $\boldsymbol{N}-\mathbf{1}$ denotes the $(N-1, N-1, \ldots)$ sequence. It is easy to see that there exists a constant $K>0$ such that

$$
K^{-1}\left(\lambda_{\left.\mathbf{i}\right|_{\mathbf{i} \wedge \mathbf{j}+\mathbf{i} \vee \mathbf{j}}}+\lambda_{\left.\mathbf{j}\right|_{\mathbf{i} \wedge \mathbf{j}+\mathbf{i} \vee \mathbf{j}}}\right) \leq|\pi(\mathbf{i})-\pi(\mathbf{j})| \leq K\left(\lambda_{\left.\mathbf{i}\right|_{\mathbf{i} \wedge \mathbf{j}+\mathbf{i} \vee \mathbf{j}}}+\lambda_{\left.\mathbf{j}\right|_{\mathbf{i} \wedge \mathbf{j}+\mathbf{i} \vee \mathbf{j}}}\right) .
$$

Hence, the distance on $[0,1]$ is not comparable with the distance on the symbolic space. More precisely, let $T$ be the set of points on the symbolic space, which has tail 0 or $N-1$, i.e. $\mathbf{i} \in T$ if and only if there exists a $k \geq 0$ such that $\sigma^{k} \mathbf{i}=\mathbf{0}$ or $\sigma^{k} \mathbf{i}=\boldsymbol{N} \mathbf{- 1}$. So if $\pi\left(\sigma^{k} \mathbf{i}\right)$ is too close to the set $\pi(T)$ infinitely often then we lose the symbolic control over the distance $\left|\pi(\mathbf{i})-\pi\left(\mathbf{i}_{n}\right)\right|$, where $\mathbf{i}_{n}$ is such that $\pi\left(\mathbf{i}_{n}\right) \rightarrow \pi(\mathbf{i})$ as $n \rightarrow \infty$.

On the other hand, the symbolic control of the set $\left\|\Pi(\mathbf{i})-\Pi\left(\mathbf{i}_{n}\right)\right\|$ is also far nontrivial. In general, $\|\Pi(\mathbf{i})-\Pi(\mathbf{j})\|=\left\|A_{\left.\mathbf{i}\right|_{\mathbf{i} \wedge \mathbf{j}}}\left(\Pi\left(\sigma^{\mathbf{i} \wedge \mathbf{j}} \mathbf{i}\right)-\Pi\left(\sigma^{\mathbf{i} \wedge \mathbf{j}} \mathbf{j}\right)\right)\right\|$ is not comparable to $\left\|A_{\mathbf{i} \mid \mathbf{i} \wedge \mathbf{j}}\right\| \cdot \| \Pi\left(\sigma^{\left.\mathbf{i} \wedge \mathbf{j}_{\mathbf{i}}\right)}-\Pi\left(\sigma^{\mathbf{i} \wedge \mathbf{j}} \mathbf{j}\right) \|\right.$, unless $\left\langle\Pi\left(\sigma^{\mathbf{i} \wedge \mathbf{j}_{\mathbf{i}}}\right)-\Pi\left(\sigma^{\mathbf{i} \wedge \mathbf{j}} \mathbf{j}\right)\right\rangle \in M$, where $M$ is the multicone satisfying the Definition 1.2. Thus, in order to handle

$$
\liminf _{n \rightarrow \infty} \frac{\log \left\|\Pi(\mathbf{i})-\Pi\left(\mathbf{i}_{n}\right)\right\|}{\log \left|\pi(\mathbf{i})-\pi\left(\mathbf{i}_{n}\right)\right|}
$$

we need that $\mathbf{i}$ is sufficiently far from the tail set $T$ and also that the points $\Pi\left(\mathbf{i}_{n}\right)$ on $\Gamma$ can be chosen such that $\left\langle\Pi\left(\sigma^{\mathbf{i} \wedge \mathbf{j}_{\mathbf{i}}}\right)-\Pi\left(\sigma^{\mathbf{i} \wedge \mathbf{i}_{n}} \mathbf{i}_{n}\right)\right\rangle \in M$. So we introduce a kind of exceptional set $B$, where both of these requirements fail. We define $B \subseteq \Sigma$ such that

$$
\begin{aligned}
& B=\{\mathbf{i} \in \Sigma: \forall n \geq 1, \forall l \geq 1, \forall m \geq 1, \exists K \geq 0, \forall k \geq K \\
& \left.\left(M\left(\Pi\left(\sigma^{k} \mathbf{i}\right)\right) \backslash B_{1 / n}\left(\Pi\left(\sigma^{k} \mathbf{i}\right)\right)\right) \cap \Gamma \backslash\left(\Gamma_{\left.\sigma^{k} \mathbf{i}\right|_{l}} \cup \Gamma_{\sigma^{k} \mathbf{i}_{l-1}\left(i_{k+l}-1\right)(N-1)^{m}} \cup \Gamma_{\left.\sigma^{k} \mathbf{i}\right|_{l-1}\left(i_{k+l}+1\right) 0^{m}}\right)=\emptyset\right\},
\end{aligned}
$$

where $\Gamma_{\bar{\imath}}=f_{\bar{\imath}}(\Gamma)$ for any finite length word $\bar{\imath} \in \Sigma^{*}$ and $M(\Pi(\mathbf{i}))$ is the cone centered at $\Pi(\mathbf{i})$. We note that if $i_{l}=0$ (or $i_{l}=N-1$ ) then we define $\Gamma_{\left.\sigma^{k} \mathbf{i}\right|_{l-1}\left(i_{l}-1\right)(N-1)^{m}}=\emptyset$ (or $\Gamma_{\left.\sigma^{k} \mathbf{i}\right|_{l-1}\left(i_{l}+1\right) 0^{m}}=\emptyset$ respectively). 


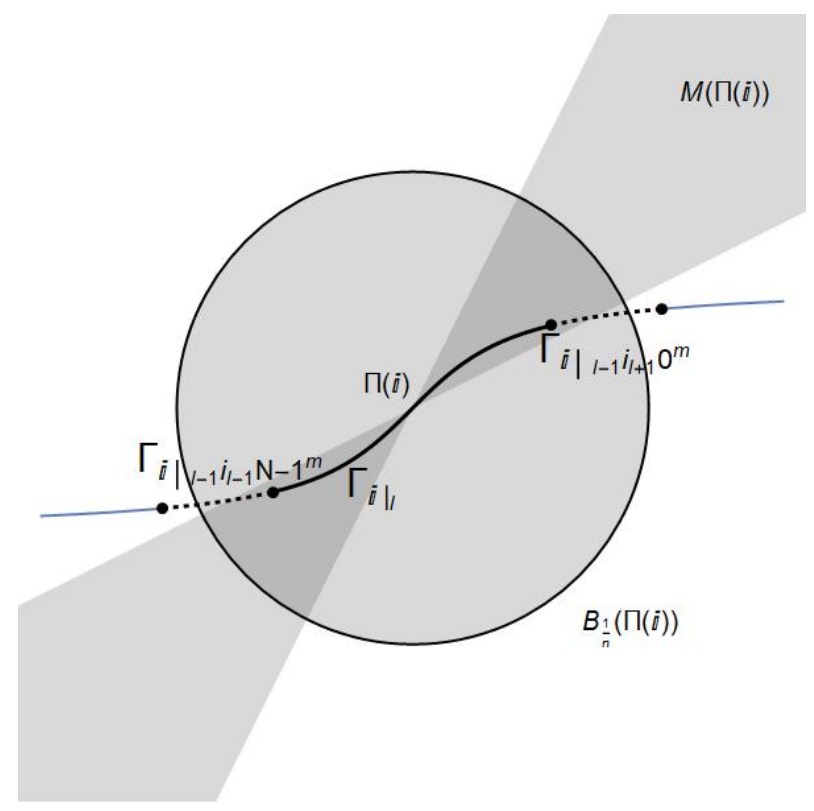

FIGURE 2. Local neighbourhood of points in $B_{n, l, m}$.

In particular, $B$ contains those points $\mathbf{i}$, for which locally the curve $\Gamma$ will leave the cone $M$ very rapidly. In other words, let

$$
\begin{aligned}
& B_{n, l, m}=\{\mathbf{i} \in \Sigma: \\
& \left.\quad\left(M(\Pi(\mathbf{i})) \backslash B_{1 / n}(\Pi(\mathbf{i}))\right) \cap \Gamma \backslash\left(\Gamma_{\left.\mathbf{i}\right|_{l}} \cup \Gamma_{\left.\mathbf{i}\right|_{l-1}\left(i_{l}-1\right)(N-1)^{m}} \cup \Gamma_{\left.\mathbf{i}\right|_{l-1}\left(i_{l}+1\right) 0^{m}}\right)=\emptyset\right\} .
\end{aligned}
$$

and

$$
B_{n, m, l, K}=\bigcap_{k=K}^{\infty} \sigma^{-k} B_{n, l, m} \text { and } B=\bigcap_{n=1}^{\infty} \bigcap_{l=0}^{\infty} \bigcap_{m=0}^{\infty} \bigcup_{K=0}^{\infty} B_{n, l, m, K} .
$$

For a visualisation of the local neighbourhood of a point in $B_{n, l, m}$, see Figure 2. In particular, we are able to handle the pointwise Hölder exponents at $\pi(\mathbf{i})$ outside of the set $B$ and we show that $B$ is small in some sense.

Lemma 3.1. Let us assume that $\mathcal{S}$ is non-degenerate. Then there exist $n \geq 1$, $l \geq 1, m \geq 1$ and $\bar{\jmath}$ finite length word with $|\bar{\jmath}|=l$, such that

$$
B_{n, l, m} \cap[\bar{\jmath}]=\emptyset .
$$

Proof. Our first claim is that there exists a finite sequence $\bar{\imath}$ such that $\left\langle A_{\bar{\imath}}\left(z_{N}-\right.\right.$ $\left.\left.z_{0}\right)\right\rangle \in M$. Suppose that this is not the case. That is, for every finite length word $\left\langle A_{\bar{\imath}}\left(z_{N}-z_{0}\right)\right\rangle \in M^{c}$. Equivalently, for every finite length word $\bar{\imath},\left\langle z_{N}-z_{0}\right\rangle \in$ $A_{\bar{\imath}}^{-1}\left(M^{c}\right)$. Thus, $\left\langle z_{N}-z_{0}\right\rangle \in \bigcap_{k=0}^{\infty} \bigcap_{|\bar{\imath}|=k} A_{\bar{\imath}}^{-1}\left(M^{c}\right)$, which contradicts to our nondegeneracy assumption.

Let us fix an $\bar{\imath}$ such that $\left\langle A_{\bar{\imath}}\left(z_{N}-z_{0}\right)\right\rangle \in M$. Then $f_{\bar{\imath}}\left(z_{N}\right) \in M\left(f_{\bar{\imath}}\left(z_{0}\right)\right)$. By continuity, one can choose $k \geq 1$ large enough such that for every $\mathbf{i} \in\left[\bar{\imath} 0^{k}\right]$,

$$
f_{\bar{\imath}}\left(z_{N}\right) \in M(\Pi(\mathbf{i}))
$$


and

$\left\|f_{\bar{\imath}}\left(z_{0}\right)-\Pi(\mathbf{i})\right\|=\left\|A_{\bar{\imath} 0^{k}}\left(z_{0}-\Pi\left(\sigma^{|\bar{\imath}|+k} \mathbf{i}\right)\right)\right\| \leq\left\|A_{\bar{\imath}}\right\|\left\|A_{0}^{k}\right\| \operatorname{diam}(\Gamma) \leq \frac{1}{2}\left\|A_{\bar{\imath}}\left(z_{N}-z_{0}\right)\right\|$, where we used the fact that $f_{0}\left(z_{0}\right)=z_{0}$. Then

$$
\left\|\Pi(\mathbf{i})-f_{\bar{\imath}}\left(z_{N}\right)\right\| \geq\left\|A_{\bar{\imath}}\left(z_{N}-z_{0}\right)\right\|-\left\|f_{\bar{\imath}}\left(z_{0}\right)-\Pi(\mathbf{i})\right\|>\frac{1}{2}\left\|A_{\bar{\imath}}\left(z_{N}-z_{0}\right)\right\| .
$$

We get that for every $\mathbf{i} \in\left[\bar{\imath} 0^{k}\right]$

$f_{\bar{\imath}}\left(z_{N}\right) \in\left(M(\Pi(\mathbf{i})) \backslash B_{\frac{1}{2}\left\|A_{\bar{\imath}}\left(z_{N}-z_{0}\right)\right\|}(\Pi(\mathbf{i}))\right) \cap \Gamma \backslash\left(\Gamma_{\bar{\imath} 0^{k}} \cup \Gamma_{\bar{\imath} 0^{k-1} 10} \cup \Gamma_{\left.\bar{\imath}\right|_{|\bar{\imath}|-1}\left(\bar{\imath}_{|\bar{\imath}|}-1\right) N}\right) \neq \emptyset$.

By fixing $\bar{\jmath}:=\bar{\imath} 0^{k}, l:=|\bar{\jmath}|, m:=1$ and $n:=\left[\frac{2}{\left\|A_{\bar{\imath}}\left(z_{N}-z_{0}\right)\right\|}\right]$, we see that $B_{n, l, m} \cap[\bar{\jmath}]=$ $\emptyset$.

Proposition 3.2. Let us assume that $\mathcal{S}$ is non-degenerate. Then $\operatorname{dim}_{P} \pi(B)<1$. Moreover, for any $\nu$ fully supported ergodic measure on $\Sigma, \nu(B)=0$.

Proof. By definition, $B_{n, l, m} \supseteq B_{n+1, l, m}, B_{n, l, m} \supseteq B_{n, l+1, m}$ and $B_{n, l, m} \supseteq B_{n, l, m+1}$. Moreover, $B_{n, l, m, K}=\sigma^{-K} B_{n, l, m, 0}$. In particular, $\sigma^{-1} B_{n, l, m, 0}=B_{n, l, m, 1} \supseteq B_{n, l, m, 0}$. Thus, for every $n \geq 1$

$$
B_{n, l, m, 0} \subseteq \bigcup_{|\bar{\imath}|=q} \varrho_{\bar{\imath}}\left(B_{n, l, m, 0}\right),
$$

where $\varrho_{\bar{\imath}}(\mathbf{i})=\bar{\imath} \mathbf{i}$. Let $n_{0} \geq 1, l_{0} \geq 1, m_{0} \geq 1$ be natural numbers and $\bar{\jmath}$ be the finite length word with $|\bar{\jmath}|=l_{0}$ as in Lemma 3.1, then

$$
B_{n_{0}, l_{0}, m_{0}, 0} \cap[\bar{\jmath}]=\bigcap_{k=0}^{\infty}\left(\sigma^{-k} B_{n_{0}, m_{0}, l_{0}} \cap[\bar{\jmath}]\right) \subseteq B_{n_{0}, m_{0}, l_{0}} \cap[\bar{\jmath}]=\emptyset .
$$

Thus,

$$
B_{n_{0}, l_{0}, m_{0}, 0} \subseteq \bigcup_{\substack{|\overline{\mid}|=l_{0} \\ \bar{\imath} \neq \bar{\jmath}}} \varrho_{\bar{\imath}}\left(B_{n_{0}, l_{0}, m_{0}, 0}\right) .
$$

Hence, $\sigma^{p} \mathbf{i} \notin[\bar{\jmath}]$ for every $\mathbf{i} \in B_{n_{0}, l_{0}, m_{0}, 0}$ and for every $p \geq 1$. Indeed, if there exists $\mathbf{i} \in B_{n_{0}, l_{0}, m_{0}, 0}$ and $p \geq 1$ such that $\sigma^{p} \mathbf{i} \in[\bar{\jmath}]$ then there exist a finite length word $\bar{\imath}$ with $|\bar{\imath}|=p$ such that $B \cap[\overline{\imath \jmath}] \neq \emptyset$. But by equations (3.5) and (3.6),

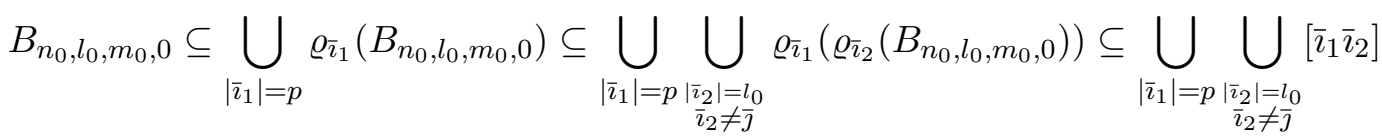

which is a contradiction. But for any fully supported ergodic measure $\nu, \nu([\bar{\jmath}])>0$ and therefore $\nu\left(B_{n_{0}, l_{0}, m_{0}, 0}\right)=0$. The second statement of the lemma follows by

$$
\nu(B) \leq \inf _{n, l, m} \nu\left(\bigcup_{K=0}^{\infty} B_{n, l, m, K}\right) \leq \sum_{K=0}^{\infty} \nu\left(B_{n_{0}, l_{0}, m_{0}, K}\right)=\sum_{K=0}^{\infty} \nu\left(B_{n_{0}, l_{0}, m_{0}, 0}\right)=0 .
$$

To prove the first assertion of the proposition, observe that by equation (3.6)

$$
\pi\left(B_{n_{0}, l_{0}, m_{0}, 0}\right) \subseteq \bigcup_{\substack{|\bar{\imath}|=l_{0} \\ \bar{\imath} \neq \bar{\jmath}}} g_{\bar{\imath}}\left(\pi\left(B_{n_{0}, l_{0}, m_{0}, 0}\right)\right)
$$


Therefore, $\pi\left(B_{n_{0}, l_{0}, m_{0}, 0}\right)$ is contained in the attractor $\Lambda$ of the IFS $\left\{g_{\bar{\imath}}\right\}_{\substack{|\overrightarrow{\mid}|=l_{0} \\ \bar{\imath} \neq \bar{j}}}$, for which $\operatorname{dim}_{B} \Lambda<1$. Hence,

$$
\operatorname{dim}_{P} \pi(B) \leq \inf _{n, l, m} \overline{\operatorname{dim}}_{B} \pi\left(B_{n, l, m, 0}\right) \leq \overline{\operatorname{dim}}_{B} \pi\left(B_{n_{0}, l_{0}, m_{0}, 0}\right) \leq \operatorname{dim}_{B} \Lambda<1 .
$$

Lemma 3.3. Let us assume that $\mathcal{S}$ is non-degenerate. Then for every $\mathbf{i} \in \Sigma \backslash B$

$$
\alpha(\pi(\mathbf{i})) \leq \limsup _{n \rightarrow+\infty} \frac{\log \left\|A_{\left.\mathbf{i}\right|_{n}}\right\|}{\log \lambda_{\left.\mathbf{i}\right|_{n}}} .
$$

Proof. Let $\mathbf{i} \in \Sigma \backslash B$. Then there exist $n \geq 1, l \geq 1, m \geq 1$ and a sequence $\left\{k_{p}\right\}_{p=1}^{\infty}$ such that $k_{p} \rightarrow \infty$ as $p \rightarrow \infty$ and

$$
\begin{aligned}
&\left(M\left(\Pi\left(\sigma^{k_{p}} \mathbf{i}\right)\right) \backslash B_{1 / n}\left(\Pi\left(\sigma^{k_{p}} \mathbf{i}\right)\right)\right) \cap \\
& \Gamma \backslash\left(\Gamma_{\left.\sigma^{k_{p}} \mathbf{i}\right|_{l}} \cup \Gamma_{\left.\sigma^{k_{p}} \mathbf{i}\right|_{l-1}\left(i_{k_{p}+l}-1\right)(N-1)^{m}} \cup \Gamma_{\left.\sigma^{k_{p}} \mathbf{i}\right|_{l-1}\left(i_{k_{p}+l}+1\right) 0^{m}}\right) \neq \emptyset
\end{aligned}
$$

Hence, there exists a sequence $\mathbf{j}_{p}$ such that $k_{p} \leq \mathbf{i} \wedge \mathbf{j}_{p} \leq k_{p}+l, \mathbf{i} \vee \mathbf{j}_{p} \leq m$,

$$
\Pi\left(\sigma^{k_{p}} \mathbf{j}_{p}\right) \in M\left(\Pi\left(\sigma^{k_{p}} \mathbf{i}\right)\right) \text { and }\left\|\Pi\left(\sigma^{k_{p}} \mathbf{j}_{p}\right)-\Pi\left(\sigma^{k_{p}} \mathbf{i}\right)\right\|>\frac{1}{n} .
$$

Thus,

$$
\begin{aligned}
& \alpha(\pi(\mathbf{i}))=\liminf _{\pi(\mathbf{j}) \rightarrow \pi(\mathbf{i})} \frac{\log \|\Pi(\mathbf{i})-\Pi(\mathbf{j})\|}{\log |\pi(\mathbf{i})-\pi(\mathbf{j})|} \leq \liminf _{p \rightarrow+\infty} \frac{\log \left\|\Pi(\mathbf{i})-\Pi\left(\mathbf{j}_{p}\right)\right\|}{\log \left|\pi(\mathbf{i})-\pi\left(\mathbf{j}_{p}\right)\right|} \\
& =\liminf _{p \rightarrow+\infty} \frac{\log \left\|A_{\left.\mathbf{i}\right|_{k_{p}}}\left(\Pi\left(\sigma^{k_{p}} \mathbf{i}\right)-\Pi\left(\sigma^{k_{p}} \mathbf{j}_{p}\right)\right)\right\|}{\log \left|\lambda_{\left.\mathbf{i}\right|_{\mathbf{i} \wedge \mathbf{j}_{p}+\mathbf{i} \vee \mathbf{j}_{p}}}\left(\pi\left(\sigma^{\mathbf{i} \wedge \mathbf{j}_{p}+\mathbf{i} \vee \mathbf{j}_{p}} \mathbf{i}\right)-\pi\left(\sigma^{\mathbf{i} \wedge \mathbf{j}_{p}+\mathbf{i} \vee \mathbf{j}_{p}} \mathbf{j}_{p}\right)\right)\right|},
\end{aligned}
$$

and by $(2.3),(3.8)$,

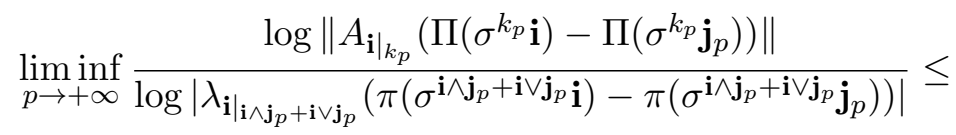

$$
\begin{aligned}
& \liminf _{p \rightarrow+\infty} \frac{\log \left(C^{-1} / n\right)+\log \left\|A_{\left.\mathbf{i}\right|_{k_{p}}}\right\|}{\log \lambda_{\left.\mathbf{i}\right|_{k_{p}}}+\log d^{\prime}} \leq \limsup _{p \rightarrow+\infty} \frac{\log \left\|A_{\left.\mathbf{i}\right|_{p}}\right\|}{\log \lambda_{\left.\mathbf{i}\right|_{p}}},
\end{aligned}
$$

where $d^{\prime}=\left(\max _{i} \lambda_{i}\right)^{m+l}$.

Lemma 3.4. Let us assume that $\mathcal{S}$ is non-degenerate. Then for every ergodic, $\sigma$ invariant, fully supported measure $\mu$ on $\Sigma$ such that $\sum_{k=0}^{\infty}\left(\mu\left[0^{k}\right]+\mu\left(\left[N^{k}\right]\right)\right.$ is finite, then

$$
\alpha(\pi(\mathbf{i}))=\lim _{n \rightarrow+\infty} \frac{\log \left\|A_{\left.\mathbf{i}\right|_{n}}\right\|}{\log \lambda_{\left.\mathbf{i}\right|_{n}}} \text { for } \mu \text {-a.e. } \mathbf{i} \in \Sigma .
$$

Proof. By Proposition 3.2, we have that $\mu(B)=0$. Thus, by Lemma 3.3, for $\mu$-a.e. i

$$
\alpha(\pi(\mathbf{i})) \leq \lim _{n \rightarrow+\infty} \frac{\log \left\|A_{\left.\mathbf{i}\right|_{n}}\right\|}{\log \lambda_{\left.\mathbf{i}\right|_{n}}} .
$$

On the other hand, for every $\mathbf{i} \in \Sigma$,

$$
\alpha(\pi(\mathbf{i}))=\liminf _{\pi(\mathbf{j}) \rightarrow \pi(\mathbf{i})} \frac{\log \|\Pi(\mathbf{i})-\Pi(\mathbf{j})\|}{\log |\pi(\mathbf{i})-\pi(\mathbf{j})|} \geq \liminf _{\mathbf{j} \rightarrow \mathbf{i}} \frac{\log \left\|A_{\left.\mathbf{i}\right|_{\mathbf{i} \wedge \mathbf{j}}}\right\|}{\log \lambda_{\left.\mathbf{i}\right|_{\mathbf{i} \wedge \mathbf{j}+\mathbf{i} \vee \mathbf{j}}}+\log \min _{i} \lambda_{i}} .
$$


Hence, to verify the statement of the lemma, it is enough to show that

$$
\lim _{\mathbf{j} \rightarrow \mathbf{i}} \frac{\log \lambda_{\left.\mathbf{i}\right|_{\mathbf{i} \wedge \mathbf{j}}}}{\log \lambda_{\left.\mathbf{i}\right|_{\mathbf{i} \wedge \mathbf{j}+\mathbf{i} \vee \mathbf{j}}}}=1 \text { for } \mu \text {-a.e. } \mathbf{i} .
$$

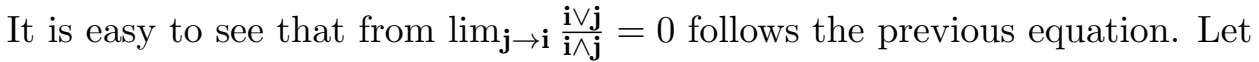

$$
R_{n}=\left\{\mathbf{i}: \exists \mathbf{j}_{k} \text { s. t. } \mathbf{j}_{k} \rightarrow \mathbf{i} \text { as } k \rightarrow \infty \text { and } \lim _{k \rightarrow \infty} \frac{\mathbf{i} \vee \mathbf{j}_{k}}{\mathbf{i} \wedge \mathbf{j}_{k}}>\frac{1}{n}\right\}
$$

In other words,

$$
R_{n}=\bigcap_{K=0}^{\infty} \bigcup_{k=K}^{\infty} \bigcup_{i_{1}, \ldots, i_{k}=0}^{N}[i_{1}, \ldots, i_{k}, \overbrace{0, \ldots, 0}^{\lfloor k / n\rfloor}] \cup[i_{1}, \ldots, i_{k}, \overbrace{N, \ldots, N}^{\lfloor k / n\rfloor}]
$$

Therefore, for any $\mu$ ergodic $\sigma$-invariant measure and for every $K \geq 0$

$$
\mu\left(R_{n}\right) \leq \sum_{k=K}^{\infty}\left(\mu\left(\left[0^{\lfloor k / n\rfloor}\right]\right)+\mu\left(\left[N^{\lfloor k / n\rfloor}\right]\right)\right) .
$$

Since by assumption the sum on the right hand side is summable, we get $\mu\left(R_{n}\right)=0$ for every $n \geq 1$.

Lemma 3.5. Let us assume that $\mathcal{S}$ is non-degenerate and satisfies (1.10). Then for every $\mathbf{i} \in \Sigma$

$$
\alpha(\pi(\mathbf{i})) \geq \liminf _{n \rightarrow+\infty} \frac{\log \left\|A_{\left.\mathbf{i}\right|_{n}}\right\|}{\log \lambda_{\left.\mathbf{i}\right|_{n}}} .
$$

Proof. Let us observe that by the zipper property $f_{i}(\Pi(\mathbf{0}))=f_{i-1}(\mathbf{N}-\mathbf{1})$ for every $1 \leq i \leq N-1$. Moreover, for any $\mathbf{i}, \mathbf{j}$ with $i_{\mathbf{i} \wedge \mathbf{j}+1}=j_{\mathbf{i} \wedge \mathbf{j}+1}+1$,

$$
\mathbf{i} \vee \mathbf{j}=\min \left\{\sigma^{\mathbf{i} \wedge \mathbf{j}+1} \mathbf{i} \wedge \mathbf{N}-\mathbf{1}, \sigma^{\mathbf{i} \wedge \mathbf{j}+1} \mathbf{j} \wedge \mathbf{0}\right\}
$$

Thus, if $i_{\mathbf{i} \wedge \mathbf{j}+1}=j_{\mathbf{i} \wedge \mathbf{j}+1}+1$

$$
\begin{aligned}
& \|\Pi(\mathbf{i})-\Pi(\mathbf{j})\|=\left\|\Pi(\mathbf{i})-\Pi\left(\mathbf{i} \mid \mathbf{i} \wedge \mathbf{j} i_{\mathbf{i} \wedge \mathbf{j}+1} \mathbf{0}\right)+\Pi\left(\left.\mathbf{j}\right|_{\mathbf{i} \wedge \mathbf{j}} j_{\mathbf{i} \wedge \mathbf{j}+1} \mathbf{N}-\mathbf{1}\right)-\Pi(\mathbf{j})\right\| \\
& =\left\|A_{\left.\mathbf{i}\right|_{\mathbf{i} \wedge \mathbf{j}+\mathbf{i} \vee \mathbf{j}}}\left(\Pi\left(\sigma^{\mathbf{i} \wedge \mathbf{j}+\mathbf{i} \vee \mathbf{j}} \mathbf{i}\right)-\Pi(\mathbf{0})\right)+A_{\left.\mathbf{i}\right|_{\mathbf{i} \wedge \mathbf{j}+\mathbf{i} \vee \mathbf{j}}}\left(\Pi(\boldsymbol{N}-\mathbf{1})-\Pi\left(\sigma^{\mathbf{i} \wedge \mathbf{j}+\mathbf{i} \vee \mathbf{j}} \mathbf{j}\right)\right)\right\| \\
& \leq\left(\left\|A_{\left.\mathbf{i}\right|_{\mathbf{i} \wedge \mathbf{j}+\mathbf{i} \vee \mathbf{j}}}\right\|+\left\|A_{\left.\mathbf{j}\right|_{\mathbf{i} \wedge \mathbf{j}+\mathbf{i} \vee \mathbf{j}}}\right\|\right) \operatorname{diam}(\Gamma) \text {. }
\end{aligned}
$$

The case $i_{\mathbf{i} \wedge \mathbf{j}+1}=j_{\mathbf{i} \wedge \mathbf{j}+1}-1$ is similar, and if $\left|i_{\mathbf{i} \wedge \mathbf{j}+1}-j_{\mathbf{i} \wedge \mathbf{j}+1}\right| \neq 1$ then $\mathbf{i} \vee \mathbf{j}=0$, so (3.9) holds trivially. Moreover by (3.3), there exist constants $K_{1}, K_{2}>0$ such that for every $\mathbf{i}, \mathbf{j} \in \Sigma$

$$
\frac{\log \|\Pi(\mathbf{i})-\Pi(\mathbf{j})\|}{\log |\pi(\mathbf{i})-\pi(\mathbf{j})|} \geq \frac{-\log K_{1}+\log \left(\left\|A_{\left.\mathbf{i}\right|_{\mathbf{i} \wedge \mathbf{j}+\mathbf{i} \vee \mathbf{j}}}\right\|+\left\|A_{\left.\mathbf{j}\right|_{\mathbf{i} \wedge \mathbf{j}+\mathbf{i} \vee \mathbf{j}}}\right\|\right)}{\log \left(\lambda_{\left.\mathbf{i}\right|_{\mathbf{i} \wedge \mathbf{j}+\mathbf{i} \vee \mathbf{j}}}+\lambda_{\left.\mathbf{j}\right|_{\mathbf{i} \wedge \mathbf{j}+\mathbf{i} \vee \mathbf{j}}}\right)+\log K_{2}} .
$$


Therefore,

$$
\begin{aligned}
& \alpha(\pi(\mathbf{i}))=\liminf _{\pi(\mathbf{j}) \rightarrow \pi(\mathbf{i})} \frac{\log \|\Pi(\mathbf{i})-\Pi(\mathbf{j})\|}{\log |\pi(\mathbf{i})-\pi(\mathbf{j})|} \\
& \geq \liminf _{\mathbf{j} \rightarrow \mathbf{i}} \frac{-\log K_{1}+\log \left(\left\|A_{\left.\mathbf{i}\right|_{\mathbf{i} \wedge \mathbf{j}+\mathbf{i} \vee \mathbf{j}}}\right\|+\left\|A_{\left.\mathbf{j}\right|_{\mathbf{i} \wedge \mathbf{j}+\mathbf{i} \vee \mathbf{j}}}\right\|\right)}{\log \left(\lambda_{\left.\mathbf{i}\right|_{\mathbf{i} \wedge \mathbf{j}+\mathbf{i} \vee \mathbf{j}}}+\lambda_{\left.\mathbf{j}\right|_{\mathbf{i} \wedge \mathbf{j}+\mathbf{i} \vee \mathbf{j}}}\right)+\log K_{2}}
\end{aligned}
$$

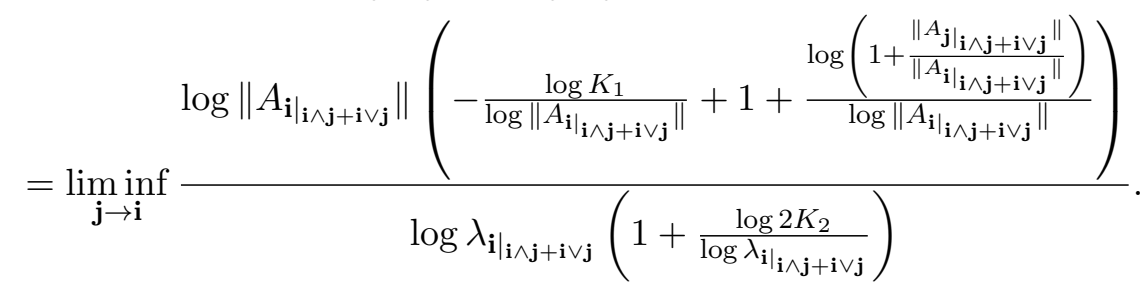

So, to verify the statement of the lemma, it is enough to show that there exists a constant $C>0$ such that for every, $\mathbf{i}, \mathbf{j} \in \Sigma$

$$
C^{-1} \leq \frac{\left\|\left.A_{\mathbf{j}}\right|_{\mathbf{i} \wedge \mathbf{j}+\mathbf{i} \vee \mathbf{j}}\right\|}{\left\|A_{\left.\mathbf{i}\right|_{\mathbf{i} \wedge \mathbf{j}+\mathbf{i} \vee \mathbf{j}}}\right\|} \leq C
$$

By Theorem 2.1(5) and (2.4), there exist $C^{\prime}>0$ such that

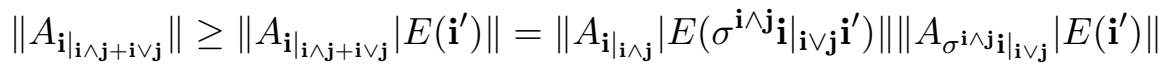

$$
\begin{aligned}
& \geq C^{\prime}\left\|A_{\left.\mathbf{i}\right|_{\mathbf{i} \wedge \mathbf{j}}}\right\|\left\|A_{\left.\sigma^{\mathbf{i} \wedge \mathbf{j}} \mathbf{j}\right|_{\mathbf{i} \vee \mathbf{j}}}\right\|
\end{aligned}
$$

and

$$
\left\|A_{\left.\mathbf{j}\right|_{\mathbf{i} \wedge \mathbf{j}+\mathbf{i} \vee \mathbf{j}}}\right\| \leq\left\|A_{\left.\mathbf{j}\right|_{\mathbf{i} \wedge \mathbf{j}}}\right\|\left\|A_{\left.\sigma^{i} \wedge \mathbf{j}\right|_{\mathbf{i} \vee \mathbf{j}}}\right\|
$$

clearly. The other bounds are similar. But if $i_{\mathbf{i} \wedge \mathbf{j}+1}=j_{\mathbf{i} \wedge \mathbf{j}+1}+1$ then $\left\|\left.A_{\sigma^{\mathbf{i} \wedge}} \mathbf{j}_{\mathbf{j}}\right|_{\mathbf{i} \backslash \mathbf{j}}\right\|=$ $\left\|A_{0}^{\mathrm{i} \vee \mathbf{j}}\right\|$ and $\left\|A_{\left.\sigma^{\mathbf{i} \wedge} \mathbf{j}_{\mathbf{i}}\right|_{\mathbf{i} \vee \mathbf{j}}}\right\|=\left\|A_{N-1}^{\mathbf{i} \vee \mathbf{j}}\right\|$. Thus, by (1.10),

$$
\alpha(\pi(\mathbf{i})) \geq \liminf _{\mathbf{j} \rightarrow \mathbf{i}} \frac{\log \left\|A_{\left.\mathbf{i}\right|_{\mathbf{i} \wedge \mathbf{j}+\mathbf{i} \vee \mathbf{j}}}\right\|}{\log \lambda_{\left.\mathbf{i}\right|_{\mathbf{i} \wedge \mathbf{j}+\mathbf{i} \vee \mathbf{j}}}} \geq \liminf _{n \rightarrow+\infty} \frac{\log \left\|A_{\left.\mathbf{i}\right|_{n}}\right\|}{\log \lambda_{\left.\mathbf{i}\right|_{n}}} .
$$

Proof of Theorem 1.3. First, we show that for $\mathcal{L}$-a.e. $x$, the local Hölder exponent is a constant. Since $\mu_{0}=\left\{\lambda_{1}, \ldots, \lambda_{N}\right\}^{\mathbb{N}}$, it is easy to see that $\pi_{*} \mu_{0}=\left.\mathcal{L}\right|_{[0,1]}$. Thus, it is enough to show that for $\mu_{0}$-a.e. $\mathbf{i} \in \Sigma, \alpha(\pi(\mathbf{i}))$ is a constant.

But by Proposition 2.4, there exists $\widehat{\alpha}$ such that for $\mu_{0}$-a.e. $\mathbf{i}$

$$
\widehat{\alpha}=\lim _{n \rightarrow+\infty} \frac{\log \left\|A_{\left.\mathbf{i}\right|_{n}}\right\|}{\log \lambda_{\left.\mathbf{i}\right|_{n}}} .
$$

By definition of Bernoulli measure, $\sum_{k=0}^{\infty} \mu_{0}\left(\left[0^{k}\right]\right)+\mu_{0}\left(\left[N^{k}\right]\right)=\frac{1}{1-\lambda_{1}}+\frac{1}{1-\lambda_{N}}$. Thus, by Lemma 3.4, $\alpha(\pi(\mathbf{i}))=\widehat{\alpha}$ for $\mu_{0}$-a.e. $\mathbf{i}$, and by Lemma 2.5 , we have $\widehat{\alpha} \geq 1 / d_{0}$.

We show now the lower bound for (1.9). By Lemma 2.3 and Proposition 2.4, the map $t \mapsto P^{\prime}(t)$ is continuous and non-increasing on $\mathbb{R}$. Hence, for every $\beta \in$ $\left(\alpha_{\min }, \alpha_{\max }\right)$ there exists a $t_{0} \in \mathbb{R}$ such that $P^{\prime}\left(t_{0}\right)=\beta$. By Proposition 2.4, there exists a $\mu_{t_{0}}$ Gibbs measure on $\Sigma$ such that

$$
\lim _{n \rightarrow+\infty} \frac{\log \left\|A_{\left.\mathbf{i}\right|_{n}}\right\|}{\log \lambda_{\left.\mathbf{i}\right|_{n}}}=\beta \text { for } \mu_{t_{0}} \text {-a.e. } \mathbf{i} \in \Sigma .
$$


It is easy to see that for any $\mathbf{i}$ and $n \geq 1, \mu_{t_{0}}\left(\left[\left.\mathbf{i}\right|_{n}\right]\right)>0$. Thus, by Lemma 3.4,

$$
\alpha(\pi(\mathbf{i}))=\beta \text { for } \mu_{t_{0}} \text {-a.e. } \mathbf{i} \in \Sigma .
$$

Observe that $\pi: \Sigma \mapsto[0,1]$ is a finite to one Lipschitz-map. Thus, by [17, Theorem 2.8, Corollary 4.16], $\operatorname{dim}_{H} \mu_{t}=\operatorname{dim}_{H} \mu_{t} \circ \pi^{-1}$ for every $t \in \mathbb{R}$. Therefore, by Proposition 2.4

$$
\begin{aligned}
\operatorname{dim}_{H}\{x \in[0,1]: \alpha(x)=\beta\} \geq \operatorname{dim}_{H} \mu_{t_{0}} \circ \pi^{-1}=t_{0} P^{\prime}\left(t_{0}\right)-P\left(t_{0}\right)= \\
t_{0} \beta-P\left(t_{0}\right) \geq \inf _{t \in \mathbb{R}}\{t \beta-P(t)\} .
\end{aligned}
$$

On the other hand, by Lemma 3.3

$$
\begin{aligned}
\operatorname{dim}_{H}\{x \in[0,1]: & \alpha(x)=\beta\} \\
& \leq \max \left\{\operatorname{dim}_{H} \pi(B), \operatorname{dim}_{H}\{\mathbf{i} \in \Sigma \backslash B: \alpha(\pi(\mathbf{i}))=\beta\}\right\} \\
& \leq \max \left\{\operatorname{dim}_{H} \pi(B), \operatorname{dim}_{H}\left\{\mathbf{i} \in \Sigma: \limsup _{n \rightarrow+\infty} \frac{\log \left\|A_{\left.\mathbf{i}\right|_{n}}\right\|}{\log \lambda_{\left.\mathbf{i}\right|_{n}}} \geq \beta\right\}\right\} \\
& \leq \max \left\{\operatorname{dim}_{H} \pi(B), \inf _{t \leq 0}\{t \beta-P(t)\}\right\},
\end{aligned}
$$

where in the last inequality we used Lemma 2.6.

By Proposition 2.4, the function $t \mapsto t P^{\prime}(t)-P(t)$ is continuous and $P(0)=-1$. By Proposition 3.2, $\operatorname{dim}_{H} \pi(B)<1$, thus, there exists an open neighbourhood of $t=0$ such that for every $t \in(-\rho, \rho), t P^{\prime}(t)-P(t)>\operatorname{dim}_{P} \pi(B)$. In other words, there exists an $\varepsilon>0$ such that $P^{\prime}(t) \in(\widehat{\alpha}-\varepsilon, \widehat{\alpha}+\varepsilon)$ for every $t \in(-\rho, \rho)$. Hence, for every $\beta \in[\widehat{\alpha}, \widehat{\alpha}+\varepsilon]$ there exists a $t_{0} \leq 0$ such that $P^{\prime}\left(t_{0}\right)=\beta$ and $\inf _{t \leq 0}\{t \beta-P(t)\}=t_{0} P^{\prime}\left(t_{0}\right)-P\left(t_{0}\right)>\operatorname{dim}_{H} \pi(B)$ which completes the proof of (1.9).

Finally, if (1.10) holds then by Lemma 3.5 and Lemma 2.6

$$
\begin{aligned}
& \operatorname{dim}_{H}\{x \in[0,1]: \alpha(x)=\beta\} \leq \\
& \quad \operatorname{dim}_{H}\left\{\mathbf{i} \in \Sigma: \liminf _{n \rightarrow+\infty} \frac{\log \left\|A_{\left.\mathbf{i}\right|_{n}}\right\|}{\log \lambda_{\left.\mathbf{i}\right|_{n}}} \leq \beta\right\} \leq \inf _{t \geq 0}\{t \beta-P(t)\},
\end{aligned}
$$

which completes the proof.

\section{Zippers with Assumption A}

Now, we turn to the case when our affine zipper satisfies the Assumption A. We will show that in fact in this case the exceptional set $B$, introduced in (3.4) is empty. That is, there are no points, in which local neighbourhood, the curve leaves the cone rapidly. First, let us introduce a natural ordering on $\Sigma^{*}$. For any $\bar{\imath}, \bar{\jmath} \in \Sigma^{*}$ with $\bar{\imath} \wedge \bar{\jmath}=m$ and $|\bar{\imath}|>m,|\bar{\jmath}|>m$, let

$$
\bar{\imath}<\bar{\jmath} \Leftrightarrow i_{m+1}<j_{m+1} .
$$

Moreover, let $Z_{1}:=\left\{z_{0}, \ldots, z_{N}\right\}$ the endpoints of the curves $f_{i}(\Gamma)$ and let $Z_{n}:=$ $\left\{f_{\bar{\imath}}\left(z_{k}\right),|\bar{\imath}|=n, k=0, \ldots, N-1\right\}$.

For simplicity, let us denote $f_{\bar{\imath}}\left(z_{0}\right)$ by $z_{\bar{\imath}}$. Observe that by the Zipper property $f_{\bar{\imath}}\left(z_{N}\right)=z_{\bar{\imath}_{|\bar{\imath}|-1}\left(i_{|\bar{\imath}|}-1\right)}$.

Proposition 4.1. Let us assume that $\mathcal{S}$ is non-degenerate and satisfies the Assumption $A$. Then $B=\emptyset$, where the set $B$ is defined in (3.4). 
Proof. It is enough to show that for every $\mathbf{i} \in \Sigma$

$$
C(\Pi(\mathbf{i})) \cap \Gamma=\Gamma,
$$

which is equivalent to show that for every $\mathbf{i}, \mathbf{j} \in \Sigma,\langle\Pi(\mathbf{i})-\Pi(\mathbf{j})\rangle \in C$.

Since $\left\langle z_{0}-z_{N}\right\rangle \in C$ and $C$ is invariant w.r.t all of the matrices then for every

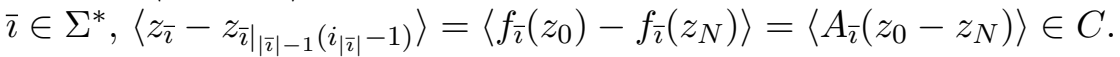

Observe by convexity of $C$, for any three vectors $x, y, w \in \mathbb{R}^{d}$, if $\langle x-y\rangle \in C$ and $\langle y-w\rangle \in C$ then $\langle x-w\rangle \in C$. Thus, by Assumption A and the convexity of the cone, for every $n \geq 1$, and for every $\bar{\imath}<\bar{\jmath} \in \Sigma$ with $|\bar{\imath}|=|\bar{\jmath}|=n,\left\langle z_{\bar{\imath}}-z_{\bar{\jmath}}\right\rangle \in C$.

Thus, for every $\mathbf{i} \neq \mathbf{j} \in \Sigma$ and for every $n \geq 1,\left\langle f_{\left.\mathbf{i}\right|_{n}}\left(z_{0}\right)-f_{\left.\mathbf{j}\right|_{n}}\left(z_{0}\right)\right\rangle=\left\langle z_{\left.\mathbf{i}\right|_{n}}-z_{\left.\mathbf{j}\right|_{n}}\right\rangle \in$ $C$. Since $C$ is closed, by taking $n$ tends to infinity, we get that $\langle\Pi(\mathbf{i})-\Pi(\mathbf{j})\rangle \in C$.

Lemma 4.2. Let us assume that $\mathcal{S}$ is non-degenerate and satisfies the Assumption $A$. Then for any $\mu$ fully supported, ergodic, $\sigma$-invariant measure on $\Sigma$

$$
\limsup _{\pi(\mathbf{j}) \rightarrow \pi(\mathbf{i})} \frac{\log \|\Pi(\mathbf{i})-\Pi(\mathbf{j})\|}{\log |\pi(\mathbf{i})-\pi(\mathbf{j})|} \leq \lim _{n \rightarrow+\infty} \frac{\log \left\|A_{\left.\mathbf{i}\right|_{n}}\right\|}{\log \lambda_{\left.\mathbf{i}\right|_{n}}} \text { for } \mu \text {-a.e. } \mathbf{i} .
$$

Proof. Observe that

$$
\begin{aligned}
& \limsup _{\pi(\mathbf{j}) \rightarrow \pi(\mathbf{i})} \frac{\log \|\Pi(\mathbf{i})-\Pi(\mathbf{j})\|}{\log |\pi(\mathbf{i})-\pi(\mathbf{j})|}= \\
& \limsup _{\pi(\mathbf{j}) \rightarrow \pi(\mathbf{i})} \frac{\log \left\|A_{\left.\mathbf{i}\right|_{\mathbf{i} \wedge \mathbf{j}+\mathbf{i} \vee \mathbf{j}}}\left(\Pi\left(\sigma^{\mathbf{i} \wedge \mathbf{j}+\mathbf{i} \vee \mathbf{j}} \mathbf{i}\right)-\Pi(\mathbf{0})\right)+A_{\left.\mathbf{j}\right|_{\mathbf{i} \wedge \mathbf{j}+\mathbf{i} \vee \mathbf{j}}}\left(\Pi(\boldsymbol{N}-\mathbf{1})-\Pi\left(\sigma^{\mathbf{i} \wedge \mathbf{j}+\mathbf{i} \vee \mathbf{j}} \mathbf{j}\right)\right)\right\|}{\left|\lambda_{\left.\mathbf{i}\right|_{\mathbf{i} \wedge \mathbf{j}}}\left(\lambda_{\left.\mathbf{i}\right|_{\mathbf{i} \vee \mathbf{j}}}\left(\pi\left(\sigma^{\mathbf{i} \wedge \mathbf{j}+\mathbf{i} \vee \mathbf{j} \mathbf{i}}\right)-0\right)+\lambda_{\left.\mathbf{j}\right|_{\mathbf{i} \vee \mathbf{j}}}\left(1-\pi\left(\sigma^{\mathbf{i} \wedge \mathbf{j}+\mathbf{i} \vee \mathbf{j}} \mathbf{j}\right)\right)\right)\right|} .
\end{aligned}
$$

By (4.1), $\left\langle\Pi\left(\sigma^{\mathbf{i} \wedge \mathbf{j}+\mathbf{i} \vee \mathbf{j}} \mathbf{i}\right)-\Pi(\mathbf{0})\right\rangle,\left\langle\Pi(N-\mathbf{1})-\Pi\left(\sigma^{\mathbf{i} \wedge \mathbf{j}+\mathbf{i} \vee \mathbf{j}} \mathbf{j}\right)\right\rangle \in C$, therefore by $(2.3)$

$$
\limsup _{\pi(\mathbf{j}) \rightarrow \pi(\mathbf{i})} \frac{\log \|\Pi(\mathbf{i})-\Pi(\mathbf{j})\|}{\log |\pi(\mathbf{i})-\pi(\mathbf{j})|} \leq \limsup _{\mathbf{j} \rightarrow \mathbf{i}} \frac{\log \left\|A_{\left.\mathbf{i}\right|_{\mathbf{i} \wedge \mathbf{j}+\mathbf{i} \vee \mathbf{j}}}\right\|+\log \left(1+\frac{\left\|A^{\mathbf{i} \vee \mathbf{j}}\right\|}{\left\|A_{N-1}^{\mathbf{i} \vee \mathbf{j}}\right\|}\right)}{\log \lambda_{\mathbf{i} \mid \mathbf{i} \wedge \mathbf{j}}} .
$$

It is easy to see that for any fully supported, ergodic, $\sigma$-invariant measure $\mu$, $\limsup _{\mathbf{j} \rightarrow \mathbf{i}} \frac{\mathbf{i} \vee \mathbf{j}}{\mathbf{i} \wedge \mathbf{j}+\mathbf{i} \vee \mathbf{j}}=0$ for $\mu$-a.e. $\mathbf{i}$. Hence, by the previous inequality, the statement follows similarly as in Lemma 3.4 .

Proof of Theorem 1.4. By Lemma 3.4 and Lemma 4.2, for every $t \in \mathbb{R}$

$$
\alpha_{r}(\pi(\mathbf{i}))=\lim _{n \rightarrow+\infty} \frac{\log \left\|A_{\left.\mathbf{i}\right|_{n}}\right\|}{\log \lambda_{\left.\mathbf{i}\right|_{n}}} \text { for } \mu_{t} \text {-a.e. } \mathbf{i} \in \Sigma .
$$

Thus, similarly to the proof of Theorem 1.3

$$
\begin{aligned}
\operatorname{dim}_{H}\left\{x \in[0,1]: \alpha_{r}(x)=\beta\right\} \geq \operatorname{dim}_{H} \mu_{t_{0}} \circ \pi^{-1}=t_{0} P^{\prime}\left(t_{0}\right)-P\left(t_{0}\right)= \\
t_{0} \beta-P\left(t_{0}\right) \geq \inf _{t \in \mathbb{R}}\{t \beta-P(t)\},
\end{aligned}
$$

where $t_{0}$ is defined such that $P^{\prime}\left(t_{0}\right)=\beta$. On the other hand,

$$
\operatorname{dim}_{H}\left\{x \in[0,1]: \alpha_{r}(x)=\beta\right\} \leq \operatorname{dim}_{H}\{x \in[0,1]: \alpha(x)=\beta\} .
$$

By Proposition 4.1, $B=\emptyset$, and similarly to the proof of Theorem 1.3,

$$
\operatorname{dim}_{H}\{x \in[0,1]: \alpha(x)=\beta\} \leq \inf _{t \leq 0}\{t \beta-P(t)\},
$$




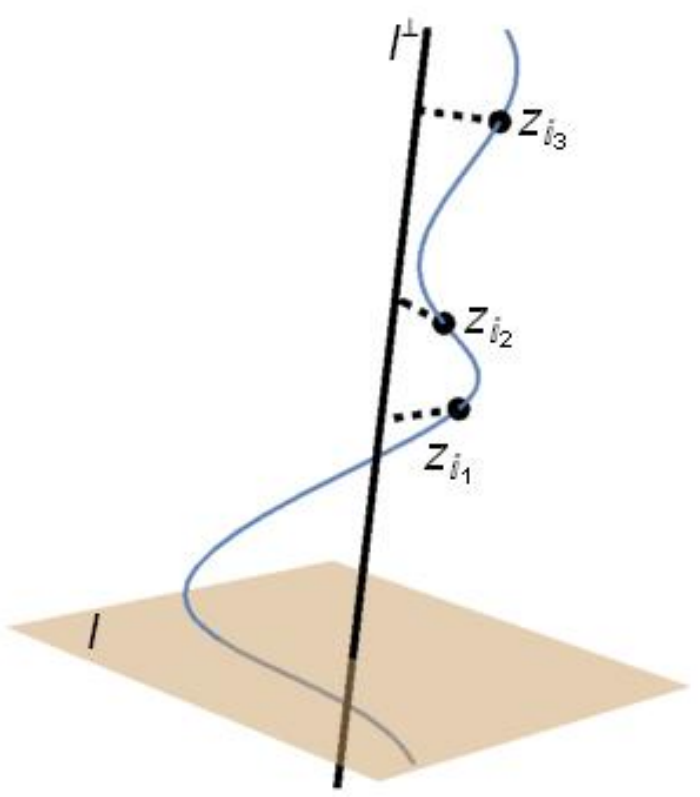

FiguRE 3 . Well ordered property of $Z_{n}$ on $l \in G(d, d-1)$.

for every $\beta \in\left[\hat{\alpha}, \alpha_{\max }\right]$. If $\Gamma$ satisfies (1.10) then by Lemma 3.5 and Lemma 2.6

$$
\operatorname{dim}_{H}\{x \in[0,1]: \alpha(x)=\beta\} \leq \inf _{t \geq 0}\{t \beta-P(t)\},
$$

which completes the proof.

Now, we turn to the equivalence of the existence of pointwise regular Hölder exponents and the Assumption A. Before that, we introduce another property and we show that in fact all of them are equivalent. Denote $\operatorname{cv}(a, b)$ open line segment in $\mathbb{R}^{d}$ connecting two points $a, b$. Moreover, let us denote the orthogonal projection to a subspace $\theta$ by $\operatorname{proj}_{\theta}$ and for a subspace $\theta$ let $\theta^{\perp}$ be the orthogonal complement of $\theta$. For a point $x$ and a subspace $\theta$, let $\theta(x)=\left\{y \in \mathbb{R}^{d}: x-y \in \theta\right\}$.

Definition 4.3. We say that $Z_{n}$ is well ordered on $l \in G(d, d-1)$ if for any $\bar{\imath}_{1}<\bar{\imath}_{2}<\bar{\imath}_{3}$

$$
\operatorname{proj}_{l^{\perp}}\left(z_{\bar{\imath}_{2}}\right) \in \operatorname{cv}\left(\operatorname{proj}_{l^{\perp}}\left(z_{\bar{\imath}_{1}}\right), \operatorname{proj}_{l^{\perp}}\left(z_{\bar{\imath}_{3}}\right)\right) .
$$

We say that $Z_{n}$ is well ordered if there exists a $\delta>0$ such that $Z_{n}$ is well ordered for all $l \in B_{\delta}(F(\Sigma))$.

Let us recall that $F: \Sigma \mapsto G(d, d-1)$ is the Hölder-continuous function defined in Theorem 2.1. So $B_{\delta}(F(\Sigma))$ is the $\delta>0$ neighbourhood of all the possible subspaces on which the growth rate of the matrices is at most the second singular value. For a visualisation of the well ordered property, see Figure 3. Roughly speaking, the well ordered property on $l \in G(d, d-1)$ means that the curve is parallel to $l^{\perp}$. The next lemma indeed verifies that the curve cannot turn back along $l^{\perp}$. 
Lemma 4.4. $Z_{n}$ is well ordered if and only if $\exists \delta>0, \forall x \in \mathbb{R}^{d}, \forall l \in B_{\delta}(F(\Sigma))$ either $\operatorname{cv}\left(z_{\bar{\imath}_{1}}, z_{\bar{\imath}_{2}}\right) \cap l(x)=\emptyset$ or $\operatorname{cv}\left(z_{\bar{\imath}_{2}}, z_{\bar{\imath}_{3}}\right) \cap l(x)=\emptyset$, for every $\bar{\imath}_{1}<\bar{\imath}_{2}<\bar{\imath}_{3}$.

Proof. Fix $\bar{\imath}_{1}<\bar{\imath}_{2}<\bar{\imath}_{3} \in\{0, \ldots, N-1\}^{n}$. Suppose that $Z_{n}$ is well ordered but for all $\delta>0$ there exists $l \in B_{\delta}(F(\Sigma))$ and $x \in \mathbb{R}^{d}$ such that $\operatorname{cv}\left(z_{\bar{\imath}_{1}}, z_{\bar{\imath}_{2}}\right) \cap l(x) \neq \emptyset$ and $\operatorname{cv}\left(z_{\bar{\imath}_{2}}, z_{\bar{\imath}_{3}}\right) \cap l(x) \neq \emptyset$. Thus,

$$
\operatorname{proj}_{l^{\perp}}(x) \in \operatorname{cv}\left(\operatorname{proj}_{l^{\perp}}\left(z_{\bar{\imath}_{2}}\right), \operatorname{proj}_{l^{\perp}}\left(z_{\bar{\imath}_{1}}\right)\right) \cap \operatorname{cv}\left(\operatorname{proj}_{l^{\perp}}\left(z_{\bar{\imath}_{2}}\right), \operatorname{proj}_{l^{\perp}}\left(z_{\bar{\imath}_{3}}\right)\right)
$$

Since the right hand side is open, and non-empty line segment, therefore

$$
\operatorname{proj}_{l^{\perp}}\left(z_{\bar{\imath}_{2}}\right) \notin \operatorname{cv}\left(\operatorname{proj}_{l^{\perp}}\left(z_{\bar{\imath}_{1}}\right), \operatorname{proj}_{l^{\perp}}\left(z_{\bar{\imath}_{3}}\right)\right),
$$

which is a contradiction.

On the other hand, suppose that $Z_{n}$ satisfy the assumption of the lemma but not well ordered. Then for every $\delta>0$ there exists an $l \in B_{\delta}(F(\Sigma))$ such that $\operatorname{proj}_{l^{\perp}}\left(z_{\bar{\imath}_{2}}\right) \notin \mathrm{cv}\left(\operatorname{proj}_{l^{\perp}}\left(z_{\bar{\imath}_{1}}\right), \operatorname{proj}_{l^{\perp}}\left(z_{\bar{\imath}_{3}}\right)\right)$. Since $B_{\delta}(F(\Sigma))$ is open, there exists an $l^{\prime} \in B_{\delta}(F(\Sigma))$ for which

$$
\operatorname{dist}\left(\operatorname{proj}_{l^{\perp \perp}}\left(z_{\bar{\imath}_{2}}\right), \operatorname{cv}\left(\operatorname{proj}_{l^{\perp \perp}}\left(z_{\bar{\imath}_{1}}\right), \operatorname{proj}_{l^{\prime \perp}}\left(z_{\bar{\imath}_{3}}\right)\right)\right)>0 .
$$

Thus, there exists $x \in \mathbb{R}^{d}$ that $\operatorname{cv}\left(z_{\bar{\imath}_{1}}, z_{\bar{\imath}_{2}}\right) \cap l^{\prime}(x) \neq \emptyset$ and $\operatorname{cv}\left(z_{\bar{\imath}_{3}}, z_{\bar{\imath}_{2}}\right) \cap l^{\prime}(x) \neq \emptyset$, which is again a contradiction.

The next lemma gives us a method to check the well ordered property.

Lemma 4.5. $Z_{0}$ is well ordered if and only if for every $n \geq 0 Z_{n}$ is well ordered.

Proof. The if part is trivial.

By definition, $Z_{n}=\bigcup_{k=0}^{N-1} f_{k}\left(Z_{n-1}\right)$. By Lemma 4.4, if $Z_{n-1}$ is well ordered then there exists $\delta>0$ such that for every $l \in B_{\delta}(F(\Sigma))$ and for every $x \in \mathbb{R}^{d}$ either $\operatorname{cv}\left(z_{\bar{\imath}_{1}}, z_{\bar{\imath}_{2}}\right) \cap l(x)=\emptyset$ or $\operatorname{cv}\left(z_{\bar{\imath}_{2}}, z_{\bar{\imath}_{3}}\right) \cap l(x)=\emptyset$. Thus, in particular for every $l \in B_{\delta}(F([k]))$. By Theorem 2.1(2), there exists $\delta^{\prime}>0$ such that $A_{k} B_{\delta}(F([k])) \supseteq$ $B_{\delta^{\prime}}(F(\Sigma))$. Thus, for every $l \in B_{\delta^{\prime}}(F(\Sigma))$ and for every $x \in \mathbb{R}^{d}$,

$$
\text { either } \operatorname{cv}\left(f_{k}\left(z_{\bar{\imath}_{1}}\right), f_{k}\left(z_{\bar{\imath}_{2}}\right)\right) \cap l(x)=\emptyset \text { or } \operatorname{cv}\left(f_{k}\left(z_{\bar{\imath}_{2}}\right), f_{k}\left(z_{\bar{\imath}_{3}}\right)\right) \cap l(x)=\emptyset
$$

for every $z_{\bar{\imath}_{1}}, z_{\bar{\imath}_{2}}, z_{\bar{\imath}_{3}} \in Z_{n-1}$ with $\bar{\imath}_{1}<\bar{\imath}_{2}<\bar{\imath}_{3}$.

Let us suppose that $Z_{n}$ is not well ordered for some $n$. Hence, there exists a minimal $n$ such that $Z_{n-1}$ is well ordered but $Z_{n}$ is not. By Lemma 4.4, for every $\delta^{\prime}>\delta>0$ there exist $\bar{\jmath}_{1}<\bar{\jmath}_{2}<\bar{\jmath}_{3} \in\{0, \ldots, N-1\}^{n+1}, l^{\prime} \in B_{\delta}(F(\Sigma))$ and $x \in \mathbb{R}^{d}$

$$
\operatorname{cv}\left(z_{\bar{\jmath}_{1}}, z_{\bar{\jmath}_{2}}\right) \cap l^{\prime}(x) \neq \emptyset \text { and } \operatorname{cv}\left(z_{\bar{\jmath}_{2}}, z_{\bar{\jmath}_{3}}\right) \cap l^{\prime}(x) \neq \emptyset .
$$

Since (4.3) holds for every $k=0, \ldots, N-1$, there are $k<m$ such that $z_{\bar{j}_{1}} \in$ $f_{k}\left(Z_{n-1}\right)$ and $z_{\bar{\jmath}_{3}} \in f_{m}\left(Z_{n-1}\right)$. On the other hand by (4.3), one of the endpoints of $f_{k}\left(Z_{n-1}\right)$ (and $f_{m}\left(Z_{n-1}\right)$ ) must be on the same side of $l^{\prime}(x)$, where $z_{\bar{\jmath}_{1}}$ (and $z_{\bar{\jmath}_{3}}$ respectively) is. Denote these endpoints by $z_{a^{\prime}}$ and $z_{b^{\prime}}$. Observe that $z_{a^{\prime}} \neq$ $z_{b^{\prime}}$. Indeed, if $z_{a^{\prime}}=z_{b^{\prime}}$ then $k=m-1$, and thus either $z_{\bar{\jmath}_{2}} \in f_{k}\left(Z_{n-1}\right)$ or $z_{\bar{\jmath}_{2}} \in f_{m}\left(Z_{n-1}\right)$. Hence, but $z_{\bar{\jmath}_{2}}$ is separated from $z_{\bar{\jmath}_{1}}, z_{\bar{\jmath}_{3}}, z_{a^{\prime}}, z_{b^{\prime}}$ by the plane $l^{\prime}(x)$, which cannot happen by (4.3).

Moreover,by (4.3), one of the endpoints of $f_{\left(\bar{\jmath}_{2}\right)_{0}}\left(Z_{n-1}\right)$ is on the same side of $l^{\prime}(x)$ with $z_{\bar{\jmath}_{2}}$, denote it by $z_{c^{\prime}}$. But the endpoints of $f_{p}\left(Z_{n-1}\right)$ are the elements of $Z_{0}$, moreover, $a^{\prime}<c^{\prime}<b^{\prime}$, which contradicts to the well ordered property of $Z_{0}$. 
Theorem 4.6. Let $\mathcal{S}$ be a non-degenerate system. Then the following three statements are equivalent

(1) $S$ satisfies Assumption A,

(2) for $\mathcal{L}$-a.e. $x, \alpha_{r}(x)$ exists,

(3) $Z_{0}$ satisfies the well-ordered property.

Proof of Theorem 4.6(1) $\Rightarrow$ Theorem 4.6(2). Similarly, to the begining of the proof of Theorem 1.3, $\pi_{*} \mu_{0}=\left.\mathcal{L}\right|_{[0,1]}$, where $\mu_{0}=\left\{\lambda_{1}, \ldots, \lambda_{N}\right\}^{\mathbb{N}}$, which is fully supported, $\sigma$-invariant, ergodic measure. Moreover, $\sum_{k=0}^{\infty} \mu_{0}\left(\left[0^{k}\right]\right)+\mu_{0}\left(\left[N^{k}\right]\right)=\frac{1}{1-\lambda_{1}}+\frac{1}{1-\lambda_{N}}$.

Thus, by Lemma 3.4

$$
\liminf _{\pi(\mathbf{j}) \rightarrow \pi(\mathbf{i})} \frac{\log \|\Pi(\mathbf{i})-\Pi(\mathbf{j})\|}{\log |\pi(\mathbf{i})-\pi(\mathbf{j})|}=\lim _{n \rightarrow+\infty} \frac{\log \left\|A_{\left.\mathbf{i}\right|_{n}}\right\|}{\log \lambda_{\left.\mathbf{i}\right|_{n}}} \text { for } \mu_{0} \text {-a.e. } \mathbf{i} \in \Sigma .
$$

But since $\mathcal{S}$ satisfies Assumption A, by using Lemma 4.2,

$$
\limsup _{\pi(\mathbf{j}) \rightarrow \pi(\mathbf{i})} \frac{\log \|\Pi(\mathbf{i})-\Pi(\mathbf{j})\|}{\log |\pi(\mathbf{i})-\pi(\mathbf{j})|} \leq \lim _{n \rightarrow+\infty} \frac{\log \left\|A_{\left.\mathbf{i}\right|_{n}}\right\|}{\log \lambda_{\left.\mathbf{i}\right|_{n}}} \text { for } \mu_{0} \text {-a.e. } \mathbf{i} \in \Sigma,
$$

which completes the proof.

Proof of Theorem 4.6(2) $\Rightarrow$ Theorem 4.6(3). Let us argue by contradiction. Assume that $\alpha_{r}(x)$ exists for $\mathcal{L}$-a.e. $x$ but there exists $Z_{n}, n \geq 0$, which does not satisfy the well-ordered property. By Lemma 4.5, $Z_{0}$ does not satisfy the well ordered property. By Lemma 4.4, let $l \in F(\Sigma), x \in \mathbb{R}^{d}$ and $z_{i-1}, z_{i}, z_{i+1} \in Z_{0}$ be such that $\operatorname{cv}\left(z_{i-1}, z_{i}\right) \cap l(x) \neq \emptyset$ and $\operatorname{cv}\left(z_{i}, z_{i+1}\right) \cap l(x) \neq \emptyset$. By continuity of the curve $\Gamma$, there exist $\mathbf{i}, \mathbf{j} \in \Sigma$ such that $i_{1}=i-1 \neq i=j_{1}, j_{2} \neq 0$ and $\Pi(\mathbf{i})-\Pi(\mathbf{j}) \in l\left(x^{\prime}\right)$ with some $x^{\prime} \in \mathbb{R}^{d}$. Hence, $\langle\Pi(\mathbf{i})-\Pi(\mathbf{j})\rangle \subset l$. By definition, there exists a $\mathbf{k} \in \Sigma$ such that $F(\mathbf{k})=l$. By using the continuity of $F: \Sigma \mapsto G(d, d-1), \Gamma$ and $\Pi: \Sigma \mapsto \Gamma$, one can choose $n, m$ sufficiently large, such that for every $\mathbf{i}^{\prime} \in\left[\left.\mathbf{i}\right|_{n}\right]$ and $\mathbf{k}^{\prime} \in\left[\left.\mathbf{k}\right|_{m}\right]$,

$$
F\left(\mathbf{k}^{\prime}\right)\left(\Pi\left(\mathbf{i}^{\prime}\right)\right) \cap \Gamma_{j_{1} j_{2}} \neq \emptyset .
$$

By ergodicity, for $\mu_{0}$-a.e. $\mathbf{i}, \sigma^{p} \mathbf{i} \in\left[k_{m}, \ldots, k_{1}, i_{1}, \ldots, i_{n}\right]$ for infinitely many $p \geq 0$, where $\left.\mathbf{k}\right|_{m}=k_{1}, \ldots, k_{m}$. Let us denote this subsequence by $p_{k}$. Let $\mathbf{k}_{k}$ be the sequence such that $\mathbf{k}_{k} \in\left[k_{1}, \ldots, k_{m}, i_{p_{k}}, \ldots, i_{1}\right]$.

By (4.4), there exists a sequence $\left\{\mathbf{j}_{k}\right\}$ such that $\mathbf{j}_{k} \wedge \mathbf{i}=p_{k}+m, \sigma^{p_{k}+m} \mathbf{j}_{k} \in\left[j_{1} j_{2}\right]$, and $\Pi\left(\sigma^{p_{k}+m} \mathbf{j}_{k}\right)-\Pi\left(\sigma^{p_{k}+m} \mathbf{i}\right) \in F\left(\mathbf{k}_{k}\right)$. By construction, $\sigma^{p_{k}+m} \mathbf{j}_{k} \wedge \sigma^{p_{k}+m_{i}} \mathbf{i}=0$ and $\sigma^{p_{k}+m} \mathbf{j}_{k} \vee \sigma^{p_{k}+m} \mathbf{i}=0$, and hence there exists a constant $c>0$ such that $\left\|\Pi\left(\sigma^{p_{k}+m} \mathbf{j}_{k}\right)-\Pi\left(\sigma^{p_{k}+m} \mathbf{i}\right)\right\|>c$. Therefore for $\mu_{0}$-a.e. $\mathbf{i}$

$$
\begin{aligned}
\alpha_{r}(\pi(\mathbf{i})) & =\lim _{\pi(\mathbf{j}) \rightarrow \pi(\mathbf{i})} \frac{\log \|\Pi(\mathbf{i})-\Pi(\mathbf{j})\|}{\log |\pi(\mathbf{i})-\pi(\mathbf{j})|}=\lim _{k \rightarrow+\infty} \frac{\log \left\|\Pi(\mathbf{i})-\Pi\left(\mathbf{j}_{k}\right)\right\|}{\log \left|\pi(\mathbf{i})-\pi\left(\mathbf{j}_{k}\right)\right|} \\
& =\lim _{k \rightarrow+\infty} \frac{\log \left\|A_{\left.\mathbf{i}\right|_{p_{k}+m}}\left(\Pi\left(\sigma^{p_{k}+m} \mathbf{i}\right)-\Pi\left(\sigma^{p_{k}+m} \mathbf{j}_{k}\right)\right)\right\|}{\log \left|\lambda_{\left.\mathbf{i}\right|_{p_{k}+m}}\left(\pi\left(\sigma^{p_{k}+m} \mathbf{i}\right)-\pi\left(\sigma^{p_{k}+m} \mathbf{j}_{k}\right)\right)\right|} \\
& \geq \lim _{k \rightarrow+\infty} \frac{\log \left\|A_{\left.\mathbf{i}\right|_{p_{k}+m}} \mid F\left(\mathbf{k}_{k}\right)\right\|}{\log \lambda_{\left.\mathbf{i}\right|_{p_{k}+m}}} \geq \lim _{k \rightarrow+\infty} \frac{\log \alpha_{2}\left(A_{\left.\mathbf{i}\right|_{p_{k}+m}}\right)}{\log \lambda_{\left.\mathbf{i}\right|_{p_{k}+m}}} \\
& \geq \frac{\log \tau}{-\chi_{\mu_{0}}}+\lim _{k \rightarrow+\infty} \frac{\log \left\|A_{\left.\mathbf{i}\right|_{p_{k}+m}}\right\|}{\log \lambda_{\left.\mathbf{i}\right|_{p_{k}+m}}}=\frac{\log \tau}{-\chi_{\mu_{0}}}+\alpha(\pi(\mathbf{i})),
\end{aligned}
$$

(2.10) where Theorem 2.1(4), Theorem 2.1(6) and Lemma 3.4. But $-\log \tau / \chi_{\mu_{0}}>0$, which is a contradiction. 
Let us recall that for any $\underline{0} \neq x \in \mathbb{R}^{d},\langle v\rangle$ denotes the unique 1-dimensional subspace in $\mathbb{P R}^{d-1}$ such that $v \in\langle v\rangle$. Also, any $V \in G(d, d-1)$ can be identified with a $d-2$ dimensional, closed submanifold $\tilde{V}$ of $\mathbb{P R}^{d-1}$ such that $\tilde{V}=\{\theta \in$ $\left.\mathbb{P R}^{d-1}: \theta \subset V\right\}$. Also, for a subset $B \subset G(d, d-1)$ we can identify it with a subset $\tilde{B}$ of $\mathbb{P R}^{d-1}$ such that $\tilde{B}=\left\{\theta \in \mathbb{P R}^{d-1}: \theta \subset V \in B\right\}$.

Proof of Theorem $4.6(3) \Rightarrow$ Theorem $4.6(1)$. Suppose that $Z_{0}$ satisfies the well ordered property. By Lemma 4.5, $Z_{n}$ satisfies the well-ordered property for every $n \geq 0$ and thus, we may assume that $\left\langle z_{\bar{\imath}}-z_{\bar{\jmath}}\right\rangle \notin \widetilde{F(\Sigma)}$ for every $z_{\bar{\imath}}, z_{\bar{\jmath}} \in Z_{n}$. Indeed, if $\left\langle z_{\bar{\imath}}-z_{\bar{\jmath}}\right\rangle \in \widetilde{F(\mathbf{i})}$ for some $\mathbf{i} \in \Sigma$ then one could find $z_{\bar{\imath}_{1}^{\prime}}, z_{\bar{\imath}^{\prime}}, z_{\bar{\imath}_{3}^{\prime}} \in Z_{n+1}$ such that $\bar{\imath}_{1}^{\prime}<\bar{\imath}_{2}^{\prime}<\bar{\imath}_{3}^{\prime}, z_{\bar{\imath}_{1}^{\prime}}=z_{\bar{\imath}}, z_{\bar{\imath}_{3}^{\prime}}=z_{\bar{\jmath}}$ and

$$
\operatorname{proj}_{F(\mathbf{i}) \perp}\left(z_{\bar{\imath}_{2}^{\prime}}\right) \notin \mathrm{cv}\left(\operatorname{proj}_{F(\mathbf{i})^{\perp}}\left(z_{\bar{\imath}_{1}^{\prime}}\right), \operatorname{proj}_{F(\mathbf{i})^{\perp}}\left(z_{\bar{\imath}_{3}^{\prime}}\right)\right) .
$$

So, for every $\bar{\imath}, \bar{\jmath} \in \Sigma^{*}$ there exists open, connected component $C_{\bar{\imath}, \bar{\jmath}}$ of $\mathbb{P R}^{d-1} \backslash$ $\widetilde{F(\Sigma)}$ such that $\left\langle z_{\bar{\imath}}-z_{\bar{\jmath}}\right\rangle \in C_{\bar{\imath}, \bar{\jmath}}$.

Then for any $\bar{\imath}_{1}<\bar{\imath}_{2}<\bar{\imath}_{3}, C_{\bar{\imath}_{1}, \bar{\imath}_{2}}=C_{\bar{\imath}_{1}, \bar{\imath}_{3}}=C_{\bar{\imath}_{2}, \bar{z}_{3}}$. Indeed, if $C_{\bar{\imath}_{1}, \bar{\tau}_{2}} \neq C_{\bar{\imath}_{1}, \bar{\imath}_{3}}$ then there exists $\widetilde{F(\mathbf{i})}$, which separates $\left\langle z_{\bar{\imath}_{2}}-z_{\bar{l}_{1}}\right\rangle$ and $\left\langle z_{\bar{\imath}_{3}}-z_{\bar{\imath}_{1}}\right\rangle$. But then, for $F(\mathbf{i})^{\perp}, \operatorname{proj}_{F(\mathbf{i}) \perp}\left(z_{\bar{\imath}_{2}}\right) \notin \mathrm{cv}\left(\operatorname{proj}_{F(\mathbf{i}) \perp}\left(z_{\bar{\imath}_{1}}\right), \operatorname{proj}_{F(\mathbf{i}) \perp}\left(z_{\bar{\imath}_{3}}\right)\right)$, which cannot happen by definition of well ordered property.

Therefore, there exists a unique open,connected component $C$ such that $\left\langle z_{\bar{\imath}}-\right.$ $\left.z_{\bar{\jmath}}\right\rangle \in C$ for every $\bar{\imath}, \bar{\jmath} \in \Sigma^{*}$. But, for any $\mathbf{i} \in \Sigma$, since $\left\langle z_{N}-z_{0}\right\rangle \notin \widetilde{F(\Sigma)}$

$$
\lim _{n \rightarrow+\infty}\left\langle A_{\left.\mathbf{i}\right|_{n}}\left(z_{N}-z_{0}\right)\right\rangle=E(\mathbf{i}),
$$

hence, $E(\Sigma) \subset C$. Thus, for any multicone $M$, for which the dominated splitting condition of index- 1 holds, the cone $M \cap C$ is invariant, i.e. $A_{i}(M \cap C) \subset M^{o} \cap C$.

On the other hand, by $\left\langle z_{N}-z_{0}\right\rangle \in C$, one can extend $M \cap C$ such that $\left\langle z_{N}-z_{0}\right\rangle \in$ $M \cap C$ and $M \cap C$ remains invariant.

\section{An example, De Rham's Curve}

In this last section of the paper, we show an application for our main theorems. The well-known de Rham's curve in $\mathbb{R}^{2}$ is the attractor of the affine zipper, formed by the functions

$$
f_{0}(x)=\left[\begin{array}{cc}
\omega & 0 \\
\omega & 1-2 \omega
\end{array}\right] x-\left[\begin{array}{c}
0 \\
2 \omega
\end{array}\right] \text { and } f_{1}(x)=\left[\begin{array}{cc}
1-2 \omega & \omega \\
0 & \omega
\end{array}\right] x+\left[\begin{array}{c}
2 \omega \\
0
\end{array}\right],
$$

where $\omega \in(0,1 / 2)$ is a parameter.

Originally, the curve was introduced and studied by de Rham [11, 12, 13] with a geometric construction. Starting from a square, it can be obtained by trisecting each side with ratios $\omega:(1-2 \omega): \omega$ and "cutting the corners" by connecting each adjacent partitioning point to get an octagon. Again, each side is divided into three parts with the same ratio and adjacent partitioning points are connected, and so on. The de Rham curve is the limit curve of this procedure. More precisely, the curve defined by the zipper in (5.1) gives the segment between two midpoints of the original square.

Let us define the following linear parametrisation of the curve. Let $v:[0,1] \mapsto \mathbb{R}^{2}$ the function of the form

$$
v(x)=f_{i}(v(2 x-i)), \text { for } x \in\left[\frac{i}{2}, \frac{i+1}{2}\right), i=0,1 .
$$


For a visualisation of a linearly parametrized de Rham curve, see Figure 4.
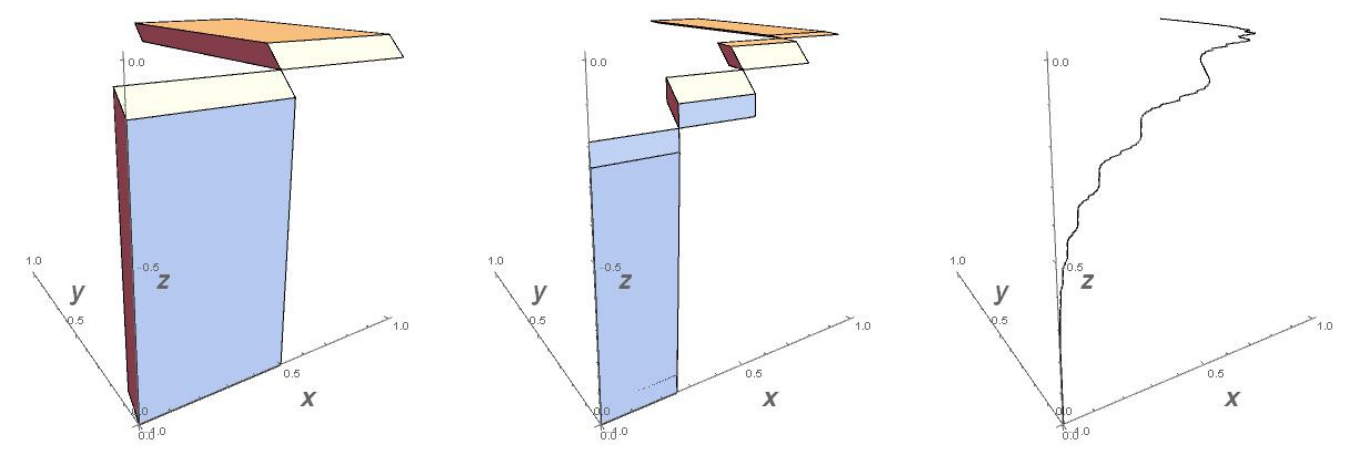

FiguRE 4. Linearly parametrized de Rham curve with parameter $\omega=1 / 10$. Left: The image of the unit cube w.r.t the IFS generating the graph of the de Rham curve. Middle: The second iteration. Right: The curve itself.

Protasov [35, 36] proved in a more general context that the set of points $x \in[0,1]$ for which $\alpha(x)=\beta$ has full measure only if $\beta=\widehat{\alpha}$, otherwise it has zero measure. Just recently, Okamura [32] bounds $\alpha(x)$ for Lebesgue typical points allowing in the definition (5.2) more than two functions and also non-linear functions under some conditions.

We show that with a suitable coordinate transform the matrices $A_{0}$ and $A_{1}$ satisfy Assumption A and hence, our results are applicable.

Lemma 5.1. For every $\omega \in(0,1 / 3) \cup(1 / 3,1 / 2)$ there exists a coordinate transform $D(\omega)$ such that $D(\omega)^{-1} A_{i} D(\omega)$ has strictly positive entries for $i=1,2$.

Proof. For $\varepsilon>0$ and $0<\delta<1$ define the coordinate transform matrices

$$
\widetilde{D}_{\varepsilon}=\left[\begin{array}{ll}
1 & \varepsilon \\
\varepsilon & 1
\end{array}\right] \text { and } \widehat{D}_{\delta}=\left[\begin{array}{cc}
1 & -\delta \\
-\delta & 1
\end{array}\right]
$$

Elementary calculations show that the matrices $\widetilde{A}_{0}=\widetilde{D}_{\varepsilon}^{-1} A_{0} \widetilde{D}_{\varepsilon}$ and $\widetilde{A}_{1}=\widetilde{D}_{\varepsilon}^{-1} A_{1} \widetilde{D}_{\varepsilon}$ have strictly positive entries whenever

$$
\frac{1}{3-\varepsilon}<\omega<\frac{1}{2-\varepsilon-\varepsilon^{2}}
$$

The largest possible interval $(1 / 3,1 / 2)$ is attained when $\varepsilon$ is arbitrarily small. Very similar calculations show that the entries of $\widehat{A}_{0}=\widehat{D}_{\delta}^{-1} A_{0} \widehat{D}_{\delta}$ and $\widehat{A}_{1}=\widehat{D}_{\delta}^{-1} A_{1} \widehat{D}_{\delta}$ are strictly positive whenever

$$
\frac{\delta}{1+3 \delta}<\omega<\frac{1}{3+\delta}
$$

which gives the open interval $(0,1 / 3)$. Also trivial calculations show that $\left\|\widetilde{A}_{i}\right\|_{1}=$ $\left\|A_{i}\right\|_{1}=\left\|\widehat{A}_{i}\right\|_{1}, i=0,1$.

Let us recall that in this case $P(t)$ has the form

$$
P(t)=\lim _{n \rightarrow+\infty} \frac{-1}{n \log 2} \log \sum_{|\bar{\imath}|=n}\left\|A_{\bar{\imath}}\right\|^{t},
$$


and

$$
\alpha_{\min }=\lim _{t \rightarrow+\infty} \frac{P(t)}{t} \text { and } \alpha_{\max }=\lim _{t \rightarrow-\infty} \frac{P(t)}{t} .
$$

Proposition 5.2. For every $\omega \in(0,1 / 4) \cup(1 / 4,1 / 3) \cup(1 / 3,1 / 2)$ the de Rham function $v:[0,1] \mapsto \mathbb{R}^{2}$, defined in (5.2), the following are true

(1) $v$ is differentiable for Lebesgue-almost every $x \in[0,1]$ with derivative vector equal to zero,

(2) Let $\mathcal{N}$ be the set of $[0,1]$ such that $v$ is not differentiable. Then $\operatorname{dim}_{H} \mathcal{N}=$ $\tau-P(\tau)>0$, where $\tau \in \mathbb{R}$ is chosen such that $P^{\prime}(\tau)=1$,

(3) $\operatorname{dim}_{H} \Pi_{*} \mu_{0}<1$, where $\mu_{0}=\left\{\frac{1}{2}, \frac{1}{2}\right\}^{\mathbb{N}}$ equidistributed measure on $\Sigma=$ $\{0,1\}^{\mathbb{N}}$ and $\Pi$ is the natural projection from $\Sigma$ to $v([0,1])$.

(4) for every $\beta \in\left[\alpha_{\min }, \alpha_{\max }\right]$

$$
\begin{aligned}
\operatorname{dim}_{H}\{x \in[0,1]: \alpha(x)=\beta\} & =\operatorname{dim}_{H}\left\{x \in[0,1]: \alpha_{r}(x)=\beta\right\} \\
& =\inf _{t \in \mathbb{R}}\{t \beta-P(t)\} .
\end{aligned}
$$

For $\omega=1 / 4$ the de Rham curve is a smooth curve, namely a parabola arc. For $\omega=1 / 3$, the matrices does not satisfy the dominated splitting condition. For this case, we refer to the work of Nikitin [31].

Proof. By Lemma 5.1, we are able to apply Theorem 1.4 and Theorem 1.5 for $\omega \neq 1 / 3$. It is easy to see that (5.1) satisfies (1.10). Thus, by Theorem 1.4, the statement (4) of the proposition follows.

On the other hand, let $\mathcal{N}$ be the set, where $v$ is not differentiable. Then

$$
\{x \in[0,1]: \alpha(x)<1\} \subseteq \mathcal{N} \subseteq\{x \in[0,1]: \alpha(x) \leq 1\} .
$$

Thus, $\operatorname{dim}_{H} \mathcal{N}=\inf _{t \in \mathbb{R}}\{t-P(t)\}$.

Now, we prove that there exists $\tau \in \mathbb{R}$ such that $P^{\prime}(\tau)=1$. Observe that $M=A_{0}+A_{1}$ is a stochastic matrix with left and right eigenvectors $p=\left(p_{1}, p_{2}\right)^{T}$ and $e=(1,1)^{T}$, respectively, corresponding to eigenvalue 1 and $p_{i}>0, p_{1}+p_{2}=1$. There exists a constant $c>0$ such that for every $\bar{\imath} \in \Sigma^{*}$

$$
c^{-1} p^{T} A_{\bar{\imath}} e \leq\left\|A_{\bar{\imath}}\right\| \leq c p^{T} A_{\bar{\imath}} e,
$$

and therefore

$$
\sum_{|\bar{\imath}|=n} p^{T} A_{\bar{\imath}} e=p^{T}\left(A_{0}+A_{1}\right)^{n} e .
$$

Thus $P(1)=0$, and

$$
\begin{aligned}
& \mu_{1}\left(\left[\left.\mathbf{i}\right|_{n}\right]\right)=p^{T} A_{\left.\mathbf{i}\right|_{n}} e=p^{T} A_{i_{1}} \ldots A_{i_{n}} e \text { and } \\
& \mu_{0}\left(\left[\left.\mathbf{i}\right|_{n}\right]\right)=\frac{1}{2^{n}} \text { for every } \mathbf{i} \in \Sigma
\end{aligned}
$$

Simple calculations show that,

$$
\mu_{1}([00])=\left(\frac{1}{2}, \frac{1}{2}\right) A_{0} A_{0}(1,1)^{T}=\omega^{2}+\frac{(1-2 \omega) \omega}{2}+\frac{(1-2 \omega)^{2}}{2},
$$

which is not equal to $1 / 4$ if $\omega \neq 1 / 4$ or $\omega \neq 1 / 2$. Thus, for $\omega \neq 1 / 4$ and $\omega \neq 1 / 2$, $\mu_{0} \neq \mu_{1}$, and by Lemma 2.5, $P^{\prime}(1)<1<P^{\prime}(0)$. Since $t \mapsto P^{\prime}(t)$ is continuous, there exists $\tau$ such that $P^{\prime}(\tau)=1$ and therefore $\operatorname{dim}_{H} \mathcal{N}=\tau-P(\tau)>0$, which completes (2). 
On the other hand, by Theorem 1.5, $\alpha_{r}(x)=P^{\prime}(0)>1$ for Lebesgue almost every $x \in[0,1]$ and therefore $v$ is differentiable with derivative vector $\underline{0}$. This implies (1).

Finally, we show statement (3) of the proposition. By using the classical result of Young

$$
\operatorname{dim}_{H} \Pi_{*} \mu_{0}=\liminf _{r \rightarrow 0+} \frac{\log \Pi_{*} \mu_{0}\left(B_{r}(x)\right)}{\log r} \text { for } \pi_{*} \mu_{0} \text {-a.e. } x \in \Gamma=v([0,1]) .
$$

For an $\mathbf{i} \in \Sigma$ and $r \in \mathbb{R}$ let $n \geq 1$ be such that $\left\|A_{\left.\mathbf{i}\right|_{n}}\right\| \leq r<\left\|A_{\left.\mathbf{i}\right|_{n-1}}\right\|$. Hence, $\Pi\left(\left[\left.\mathbf{i}\right|_{n}\right]\right) \subseteq B_{r}(\Pi(\mathbf{i}))$ and

$$
\liminf _{r \rightarrow 0+} \frac{\log \Pi_{*} \mu_{0}\left(B_{r}(\Pi(\mathbf{i}))\right)}{\log r} \leq \liminf _{n \rightarrow \infty} \frac{\log \mathbb{P}\left(\left[\left.\mathbf{i}\right|_{n}\right]\right)}{\log \left\|A_{\left.\mathbf{i}\right|_{n}}\right\|}
$$

Since $\mu_{0}([\mathbf{i} \mid n])=1 / 2^{n}$, by Proposition 2.4

$$
\liminf _{n \rightarrow \infty} \frac{\log \mathbb{P}\left(\left[\left.\mathbf{i}\right|_{n}\right]\right)}{\log \left\|A_{\left.\mathbf{i}\right|_{n}}\right\|}=\frac{1}{P^{\prime}(0)}<1 .
$$

Remark 5.3. Finally, we remark that in case of general signature vector, one may modify the definition of $\mathbf{i} \vee \mathbf{j}$ to

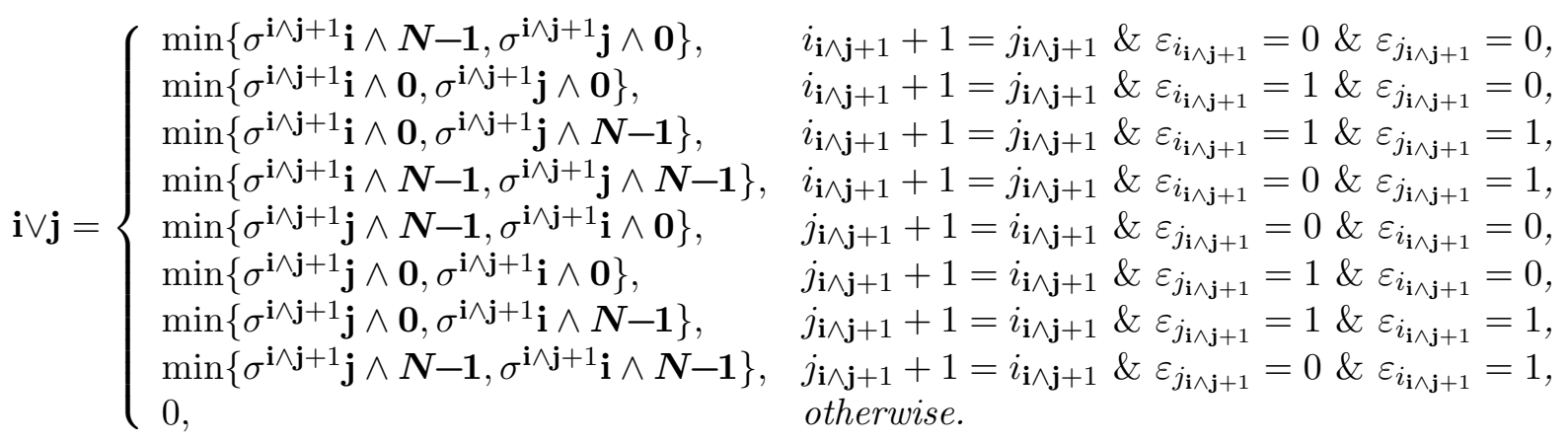

Acknowledgement. The authors would like to express their gratitude to the referee for the useful comments and suggestions and the careful reading. Bárány was partially supported by the grants OTKA K104745, EPSRC EP/J013560/1 and János Bolyai Research Scholarship of the Hungarian Academy of Sciences. Kiss was partially supported by the grant OTKA K104178 and the internal research project F1R-MTH-PUL-15MRO3 of the University of Luxembourg. Kolossváry was partially supported by the Hungarian National Eötvös Scholarship.

\section{REFERENCES}

[1] J. Aouidi and M. B. Slimane. Multi-fractal formalism for non-self-similar functions. Integral Transforms Spec. Funct., 15(3):189-207, 2004.

[2] C. Bandt and A. Kravchenko. Differentiability of fractal curves. Nonlinearity, 24(10):2717, 2011.

[3] M. F. Barnsley. Fractal functions and interpolation. Constructive Approximation, 2(1):303329, 1986.

[4] J. Barral and S. Seuret. From multifractal measures to multifractal wavelet series. J. Fourier Anal. Appl., 11(5):589-614, 2005.

[5] M. Ben Sliman. The thermodynamic formalism for the de Rham function: the increment method. Izv. Ross. Akad. Nauk Ser. Mat., 76(3):3-18, 2012. 
[6] M. Ben Slimane. Multifractal formalism for self-similar functions expanded in singular basis. Appl. Comput. Harmon. Anal., 11(3):387-419, 2001.

[7] M. Ben Slimane. Some functional equations revisited: the multifractal properties. Integral Transforms Spec. Funct., 14(4):333-348, 2003.

[8] J. Bochi and N. Gourmelon. Some characterizations of domination. Mathematische Zeitschrift, 263(1):221-231, 2009.

[9] R. Bowen. Equilibrium states and the ergodic theory of Anosov diffeomorphisms, volume 470 of Lecture Notes in Mathematics. Springer-Verlag, Berlin, 2008.

[10] C. Coiffard, C. Melot, and W. Thomas. A family of functions with two different spectra of singularities. J. Fourier Anal. Appl., 20(5):961-984,, 2014.

[11] G. de Rham. Une peu de mathmatiques propos d'une courbe plane. Elemente der Math., 2(4):73-76, 89-97, 1947.

[12] G. de Rham. Sur une courbe plane. J. Math. Pures Appl., 35:25-42, 1956.

[13] G. de Rham. Sur les courbes limits de polygones obtenus par trisection. Enseignement Math., 5:29-43, 1959.

[14] K. J. Falconer. The Hausdorff dimension of self-affine fractals. Math. Proc. Cambridge Philos. Soc., 103(2):339-350, 1988.

[15] K. J. Falconer. Fractal Geometry: Mathematical Foundations and Applications. Wiley, 1990.

[16] D.-J. Feng. Lyapunov exponents for products of matrices and multifractal analysis. Part i: Positive matrices. Israel Journal of Mathematics, 138(1):353-376, 2003.

[17] D.-J. Feng and H. Hu. Dimension theory of iterated function systems. Communications on Pure and Applied Mathematics, 62(11):1435-1500, 2009.

[18] D.-J. Feng and A. Käenmäki. Self-affine sets in analytic curves and algebraic surfaces. Ann. Acad. Sci. Fenn. Math., 2017. to appear, available at arXiv:1612.07136.

[19] D.-J. Feng and K.-S. Lau. The pressure function for products of non-negative matrices. Math. Research Letter, (9):363-378, 2002.

[20] D.-J. Feng and P. Shmerkin. Non-conformal repellers and the continuity of pressure for matrix cocycles. P. Geom. Funct. Anal., 24(4):1101-1128, 2014.

[21] Y. Heurteaux. Estimations de la dimension inférieure et de la dimension supérieure des mesures. Ann. Inst. H. Poincaré Probab. Statist., 34(3):309-338, 1998.

[22] J. E. Hutchinson. Fractals and self-similarity. Indiana Univ. Math. J., 30(5):713-747, 1981.

[23] J. Jaerisch and H. Sumi. Pointwise Hölder exponents of the complex analogues of the Takagi function in random complex dynamics". Advances in Mathematics, 313(Supplement C):839 $874,2017$.

[24] S. Jaffard. Multifractal formalism for functions. I. Results valid for all functions. SIAM J. Math. Anal., 28(4):944-970, 1997.

[25] S. Jaffard. Multifractal formalism for functions. II. Self-similar functions. SIAM J. Math. Anal., 28(4):971-998, 1997.

[26] T. Jordan, M. Pollicott, and K. Simon. Hausdorff dimension for randomly perturbed self affine attractors. Comm. Math. Phys., 270(2):519-544, 2007.

[27] A. Käenmäki. On natural invariant measures on generalised iterated function systems. Ann. Acad. Sci. Fenn. Math., 29(2):419-458, 2004.

[28] F. Ledrappier. On the dimension of some graphs. In Symbolic dynamics and its applications (New Haven, CT, 1991), volume 135 of Contemp. Math., pages 285-293. Amer. Math. Soc., Providence, RI, 1992.

[29] I. D. Morris. An inequality for the matrix pressure function and applications. Advances in Mathematics, 302:280-308, 2016.

[30] I. D. Morris. On Falconer's formula for the generalised Rényi dimension of a self-affine measure. Ann. Acad. Sci. Fenn. Math., 42(1):227-238, 2017.

[31] P. Nikitin. The Hausdorff dimension of the harmonic measure on de Rham's curve. Journal of Mathematical Sciences, 121(3):2409-2418, 2004.

[32] K. Okamura. On regularity for de Rham's functional equations. Aequationes mathematicae, 90(6):1071-1085, Dec 2016.

[33] A. Otani. Fractal dimensions of graph of Weierstrass-type function and local Hölder exponent spectra. ArXiv e-prints, Mar. 2016.

[34] M. Pollicott and P. Vytnova. Estimating singularity dimension. Math. Proc. Camb. Phil. Soc., 158(02):223-238, 2015. 
[35] V. Y. Protasov. On the regularity of de Rham curves. Izvestiya: Mathematics, 68(3):567, 2004

[36] V. Y. Protasov. Fractal curves and wavelets. Izvestiya: Mathematics, 70(5):975, 2006.

[37] V. Y. Protasov and N. Guglielmi. Matrix approach to the global and local regularity of wavelets. Poincare J. Anal. Appl., (2):77-92, 2015.

[38] K. Saka. Scaling exponents of self-similar functions and wavelet analysis. Proc. Amer. Math. Soc., 133(4):1035-1045, 2005.

[39] S. Seuret. Multifractal Analysis and Wavelets, pages 19-65. Springer International Publishing, Cham, 2016.

[40] B. Solomyak. Measure and dimension for some fractal families. Math. Proc. Cambridge Philos. Soc., 124(3):531-546, 1998

Balázs Bárány, Budapest University of Technology and Economics, MTA-BME Stochastics Research Group, P.O.Box 91, 1521 Budapest, Hungary

Mathematics Institute, University of Warwick, Coventry CV4 7AL, UK

E-mail address: balubsheep@gmail.com

Gergely Kiss, Budapest University of Technology and Economics, MTA-BMe Stochastics Research Group, P.O.Box 91, 1521 Budapest, Hungary

Faculty of Science, University of Luxembourg, Luxembourg

E-mail address: kigergo57@gmail.com

István Kolossváry, Budapest University of Technology and Economics, Institute of Mathematics, Department of Stochastics;

MTA Alfréd RÉNyi Institute of Mathematics

E-mail address: istvanko@math.bme.hu 